\title{
First and higher order uniform dual ergodic theorems for dynamical systems with infinite measure
}

\author{
Ian Melbourne $^{*} \quad$ Dalia Terhesiu ${ }^{\dagger}$
}

25 May 2011

\begin{abstract}
We generalize the proof of Karamata's Theorem by the method of approximation by polynomials to the operator case. As a consequence, we offer a simple proof of uniform dual ergodicity for a very large class of dynamical systems with infinite measure, and we obtain bounds on the convergence rate.

In many cases of interest, including the Pomeau-Manneville family of intermittency maps, the estimates obtained through real Tauberian remainder theory are very weak. Building on the techniques of complex Tauberian remainder theory, we develop a method that provides second (and higher) order asymptotics. In the process, we derive a higher order Tauberian theorem for scalar power series, which to our knowledge, has not previously been covered.
\end{abstract}

\section{$1 \quad$ Introduction and main results}

Suppose that $(X, \mu)$ is an infinite measure space and $f: X \rightarrow X$ is a conservative measure preserving transformation with transfer operator $L: L^{1}(X) \rightarrow L^{1}(X)$. The transformation $f$ is pointwise dual ergodic if there exists a positive sequence $a_{n}$ such that $a_{n}^{-1} \sum_{j=0}^{n-1} L^{j} v \rightarrow \int_{X} v d \mu$ a.e. as $n \rightarrow \infty$, for all $v \in L^{1}(X)$. If furthermore, there exists $Y \subset X$ with $\mu(Y) \in(0, \infty)$ such that $a_{n}^{-1} \sum_{j=0}^{n-1} L^{j} 1_{Y} \rightarrow \mu(Y)$ uniformly on $Y$, then $Y$ is referred to as a Darling-Kac set (see Aaronson [2] for further background) and we refer to $f$ as uniformly dual ergodic. At present, it is an open question whether every pointwise dual ergodic transformation has a Darling-Kac set. However, it is desirable to prove pointwise dual ergodicity by identifying Darling-Kac sets, as this facilitates the proof of several strong properties for $f$ (see for instance [1, 2, 3, 5, 6, 38, 40, 42] and for the setting of Markov chains [8, 10, 29]; see also [9] and references therein).

\footnotetext{
*Department of Mathematics, University of Surrey, Guildford, Surrey GU2 7XH, UK
}

${ }^{\dagger}$ Department of Mathematics, University of Surrey, Guildford, Surrey GU2 7XH, UK 


\subsection{Uniform dual ergodicity}

An important class of examples is provided by interval maps with indifferent fixed points (see for instance [37, 38, 42]). Standard examples are the family of PomeauManneville intermittency maps [34] which are uniformly expanding except for an indifferent fixed point at 0 . To fix notation, we focus on the version studied by Liverani et al. [30]:

$$
f x=\left\{\begin{array}{ll}
x\left(1+2^{\alpha} x^{\alpha}\right), & 0<x<\frac{1}{2} \\
2 x-1, & \frac{1}{2}<x<1
\end{array} .\right.
$$

It is well known that for $\alpha \geq 1$, we are in the situation of infinite ergodic theory: there exist a unique (up to scaling) $\sigma$-finite, absolutely continuous invariant measure $\mu$, and $\mu([0,1])=\infty$. Let $\beta=1 / \alpha$. Using the standard procedure of inducing, several studies established that $Y=\left[\frac{1}{2}, 1\right]$ is a Darling-Kac set for $f$ with return sequence $a_{n}$ proportional to $n^{\beta}$ for $\beta \in(0,1)$, and proportional to $n / \log n$ for $\beta=1$.

An important refinement is the limit theorem of Thaler [38] where the convergence of $a_{n}^{-1} \sum_{j=1}^{n} L^{j} v$ is shown to be uniform on compact subsets of $(0,1]$ for all observables of the form $v=u / h$ where $u$ is Riemann integrable and $h$ is the density.

The results of [38] are formulated for Markov maps of the interval with indifferent fixed points. This includes transformations with slowly varying return sequences, the so called $\beta=0$ case. One such example is

$$
f x=\left\{\begin{array}{ll}
x\left(1+x e^{-1 / x}\right), & 0<x<\frac{1}{2} \\
2 x-1, & \frac{1}{2}<x<1
\end{array},\right.
$$

with return sequence $a_{n}$ proportional to $\log n$ (see [37]). Zweimüller [41, 42] relaxed the Markov condition and systematically studied non-Markovian nonuniformly expanding interval maps (so-called AFN maps). In particular, 41] obtained a spectral decomposition into basic (conservative and ergodic) sets and proved that for each basic set there is a $\sigma$-finite absolutely continuous invariant measure, unique up to scaling. The results in [38] on uniform dual ergodicity were extended in [42] to the class of AFN maps.

In this paper, we generalize the proof of Karamata's Theorem by the elementary method of approximation by polynomials [24, 25] to the operator case. As a consequence, we offer a simple proof of uniform dual ergodicity for a large class of dynamical systems with infinite measure. This method of proof, combined with techniques from [31], allows us to strengthen the results in [38, 42].

It is convenient to describe our first result in the setting of AFN maps $f: X \rightarrow X$, though it applies to much more general systems, as described in Section 3. Let $X^{\prime} \subset X$ denote the complement of the indifferent fixed points. For any compact subset $A \subset X^{\prime}$, the construction in [41] yields a suitable first return set $Y$ containing $A$. Fix such a set $Y$ with first return time function $\varphi: Y \rightarrow \mathbb{Z}^{+}, \varphi(y)=\inf \left\{n \geq 1: f^{n} y \in Y\right\}$. 
We assume that the tail probabilities are regularly varying: $\mu(y \in Y: \varphi(y)>n)=$ $\ell(n) n^{-\beta}$ where $\ell$ is slowly varying and $\beta \in[0,1]$.

For (1.1), $\ell(n)$ is asymptotically constant and $\beta=\frac{1}{\alpha}$. For (1.2), $\ell(n)$ is asymptotically proportional to $1 / \log n$ and $\beta=0$.

Define $m(n)=\ell(n)$ for $\beta \in[0,1)$ and $m(n)=\tilde{\ell}(n)=\sum_{j=1}^{n} \ell(j) j^{-1}$ for $\beta=1$. Set $D_{\beta}=\Gamma(1-\beta) \Gamma(1+\beta)$ for $\beta \in(0,1)$ and $D_{0}=D_{1}=1$.

Theorem 1.1 Suppose that $f: X \rightarrow X$ is an AFN map with regularly varying tail probabilities, $\beta \in[0,1]$. Consider observables of the form $v=\xi u$ where $\xi$ is $\mu$-integrable and bounded variation on $X$, and $u$ is Riemann integrable. Then

$$
\lim _{n \rightarrow \infty} a_{n}^{-1} \sum_{j=0}^{n-1} L^{j} v=\int_{X} v d \mu
$$

uniformly on compact subsets of $X^{\prime}$, where $a_{n}=D_{\beta}^{-1} n^{\beta} m(n)^{-1}$.

The proof of Theorem 1.1 is provided in Subsection 3.2.

Remark 1.2 Even in the case of AFN maps, the class of observables $v$ is much larger than in [38, 42. We note that Theorem 1.1] follows from our earlier paper [31] when $\beta \in\left(\frac{1}{2}, 1\right]$, but that the methods in [31] fail for $\beta \in\left[0, \frac{1}{2}\right]$.

\subsection{Convergence rates - real Tauberian theory}

Karamata's approximation by polynomials method (generalized to the operator case) allows us to obtain convergence rates in Theorem 1.1 by mimicking the arguments used in real Tauberian remainder theory (see for instance [28, Chapter VII] and references therein). Below, we provide an example of such a theorem, restricting to the case of (1.2), where the remainder is optimal. More general examples of Tauberian theorems with remainders for positive operators are covered in Section 3.3. In particular, Theorem 3.2(b) provides sharp remainders for a large class of dynamical systems in the $\beta=0$ case.

Theorem 1.3 Let $f$ be defined by (1.2) and let $h$ be the density for $\mu$. Set $c=\frac{1}{2} h\left(\frac{1}{2}\right)$. Suppose that $v:[0,1] \rightarrow \mathbb{R}$ is Hölder or bounded variation supported on a compact subset of $(0,1]$. Then

$$
c \sum_{j=0}^{n-1} L^{j} v=\log n \int_{0}^{1} v d \mu+O(1)
$$

uniformly on compact sets of $(0,1]$. 
Theorem 1.3 is proved in Section 3 .

In the situation of (1.1), we obtain the error term $O\left(n^{\beta} / \log n\right.$ ) (see Theorem $3.2(\mathrm{a})$ ). The remainder in this case is not optimal (cf. [31, Corollary 9.3]). However, this result is probably the best possible using methods from real Tauberian theory, as discussed in Remark 3.8. Hence in the next subsection we turn to the complex theory.

\subsection{Higher order asymptotics - complex Tauberian theory}

Building on the techniques of complex remainder theory for the scalar case (see Korevaar [28, Chapter III.16]), we develop a method that provides higher order uniform dual ergodic theorems for infinite measure preserving systems. For simplicity, in this paper we focus on the typical case of (1.1), but as explained in the sequel, our method applies to other cases of interest. Also, the case $\beta=1$ of (1.1) has been fully understood in [31]; more general examples are considered in work in progress. Hence higher order theory for the case of $\beta=1$ is not considered in this paper.

Theorem 1.4 Let $f$ be defined by (1.1) with $\beta \in(0,1)$. Suppose that $v:[0,1] \rightarrow \mathbb{R}$ is Hölder or bounded variation supported on a compact subset of $(0,1]$.

Set $k=\max \{j \geq 0:(j+1) \beta-j>0\}$. Then for any $\epsilon>0$,

$$
\sum_{j=0}^{n-1} L^{j} v=\left(C_{0} n^{\beta}+C_{1} n^{2 \beta-1}+C_{2} n^{3 \beta-2}+\cdots+C_{k} n^{(k+1) \beta-k}\right) \int_{0}^{1} v d \mu+O\left(n^{\epsilon}\right),
$$

uniformly on compact subsets of $(0,1]$, where $C_{1}, C_{2}, \ldots$ are real nonzero constants (depending only on $f$ ).

Theorem 1.4 is proved in Section 4 .

Remark 1.5 In particular, when $\beta>\frac{1}{2}$ we obtain the second order asymptotics $\lim _{n \rightarrow \infty} n^{1-\beta}\left(n^{-\beta} \sum_{j=0}^{n-1} L^{j} v-C_{0} \int_{0}^{1} v d \mu\right)=C_{1} \int_{0}^{1} v d \mu$.

Remark 1.6 In [31], we obtained first and higher order asymptotics of the iterates $L^{n}$ but the methods require $\beta>\frac{1}{2}$. As a byproduct, we obtained a weakened version of Theorem 1.4 with $n^{\epsilon}$ replaced by $n^{\frac{1}{2}}$. In particular, the second order asymptotics result in Remark 1.5 is contained in [31. only for $\beta>\frac{3}{4}$.

In the process of proving Theorem 1.4, we obtain the following complex Tauberian theorem with remainder, which to our knowledge has not been previously considered.

Theorem 1.7 Let $\Phi(z)=\sum_{j=0}^{\infty} u_{j} z^{j}$ be a convergent power series for $|z|<1$ with $\left|u_{j}\right|=O(1)$. Let $1>\gamma_{1}>\gamma_{2}>\cdots>\gamma_{k}>0$, where $k \geq 0$. Write $z=e^{-u+i \theta}, u>0$, 
$\theta \in[0,2 \pi)$. Suppose that

$$
\Phi(z)=\sum_{r=1}^{k} A_{r}(u-i \theta)^{-\gamma_{r}}+O(1), \text { as } u, \theta \rightarrow 0
$$

for $A_{1}, \ldots, A_{k}$ real constants. Then for any $\epsilon>0$,

$$
\sum_{j=0}^{n-1} u_{j}=\sum_{r=1}^{k} \frac{A_{r}}{\Gamma\left(1+\gamma_{r}\right)} n^{\gamma_{r}}+O\left(n^{\epsilon}\right) .
$$

\subsection{Renewal sequences}

Theorem 1.7 has immediate applications to scalar renewal sequences with infinite mean. To make this explicit we recall some basic background on scalar renewal theory. For more details we refer the reader to [12, 9]. Let $\left(X_{i}\right)_{i \geq 1}$ be a sequence of positive integer-valued independent identically distributed random variables with probabilities $P\left(X_{i}=j\right)=f_{j}$. Define the partial sums $S_{n}=\sum_{j=1}^{n} X_{j}$, and set $u_{0}=1$ and $u_{n}=\sum_{j=1}^{n} f_{j} u_{n-j}, n \geq 1$. Then it is easy to see that $u_{n}=\sum_{j=0}^{n} P\left(S_{j}=n\right)$. The sequences $\left(u_{n}\right)_{n \geq 0}$ are called renewal sequences.

The analysis of scalar renewal sequences with infinite mean relies crucially on the assumption of regularly varying tails: $\sum_{j>n} f_{j}=\ell(n) n^{-\beta}$, where $\ell$ is slowly varying and $\beta \in[0,1]$ (see [12, 9] and references therein). Then Karamata's Tauberian theorem yields $\sum_{j=0}^{n} u_{j} \sim D_{\beta}^{-1} n^{\beta} m^{-1}(n)$, where $D_{\beta}$ and $m(n)$ are as defined above. (For results on first order asymptotics for $u_{n}$ we refer to [9, 11, 14. )

A natural problem is to consider higher order expansions of $\sum_{j=0}^{n} u_{j}$. Suppose for example that $\sum_{j>n} f_{j}=c n^{-\beta}+b(n)+c(n)$, where $b(n)=O\left(n^{-2 \beta}\right)$ and $c(n)$ is summable. If $\beta \leq 1 / 2$, assume further that $b(n)$ is monotone. Set $k=\max \{j \geq 0$ : $(j+1) \beta-j>0\}$. It follows from the methods in this paper (specifically Theorem 1.7 together with a scalar version of Lemma 2.5), that for any $\epsilon>0$,

$$
\sum_{j=0}^{n} u_{j}=\left(C_{0} n^{\beta}+C_{1} n^{2 \beta-1}+C_{2} n^{3 \beta-2}+\cdots+C_{k} n^{(k+1) \beta-k}\right)+O\left(n^{\epsilon}\right),
$$

where $C_{1}, C_{2}, \ldots$ are real nonzero constants.

Our method for proving uniform dual ergodic theorems centres around an operator version of renewal sequences. Let $(X, \mu)$ be a measure space (finite or infinite), and $f: X \rightarrow X$ a conservative measure preserving map. Fix $Y \subset X$ with $\mu(Y) \in(0, \infty)$. Let $\varphi: Y \rightarrow \mathbb{Z}^{+}$be the first return time $\varphi(y)=\inf \left\{n \geq 1: f^{n} y \in Y\right\}$ (finite almost everywhere by conservativity). Let $L: L^{1}(X) \rightarrow L^{1}(X)$ denote the transfer operator for $f$ and define

$$
T_{n}=1_{Y} L^{n} 1_{Y}, \quad n \geq 0, \quad R_{n}=1_{Y} L^{n} 1_{\{\varphi=n\}}, \quad n \geq 1 .
$$


Thus $T_{n}$ corresponds to $u_{n}$ (returns to $Y$ ) and $R_{n}$ corresponds to $f_{n}$ (first returns). The relationship $T_{n}=\sum_{j=1}^{n} T_{n-j} R_{j}$ generalises the notion of scalar renewal sequences.

Operator renewal sequences were introduced by Sarig [35] to study lower bounds for mixing rates associated with finite measure preserving systems, and this technique was substantially extended and refined by Gouëzel [15, 16]. The authors [31] developed a theory of renewal operator sequences for dynamical systems with infinite measure, generalizing the results of [14, 11] to the operator case and obtaining mixing rates for a large class of systems including (1.1) for $\beta \in\left(\frac{1}{2}, 1\right]$. The uniform dual ergodic theorems proved in this paper follow from an operator version of Theorem 1.7, namely Theorem 4.1.

The rest of the paper is organised as follows. In Section 2, we describe the general framework for our results on the renewal operators $T_{n}$. In Section 3 , we generalize the proof of Karamata's Theorem by the elementary method of approximation by polynomials and obtain uniform dual ergodic theorems with remainders for a large class of dynamical systems with infinite measure. Section 4 is devoted to higher order uniform dual ergodic theorems for a large class of systems. Appendix A contains the proof of several technical results stated in Section 2. Appendix B contains computations of some complex contour integrals. In Appendix C, we provide explicit tail probabilities for (1.1) and (1.2).

Notation We use "big O" and « notation interchangeably, writing $a_{n}=O\left(b_{n}\right)$ or $a_{n} \ll b_{n}$ as $n \rightarrow \infty$ if there is a constant $C>0$ such that $a_{n} \leq C b_{n}$ for all $n \geq 1$.

\section{General framework}

Let $(X, \mu)$ be an infinite measure space, and $f: X \rightarrow X$ a conservative measure preserving map. Fix $Y \subset X$ with $\mu(Y)=1$. Let $\varphi: Y \rightarrow \mathbb{Z}^{+}$be the first return time $\varphi(y)=\inf \left\{n \geq 1: f^{n} y \in Y\right\}$ and define the first return map $F=f^{\varphi}: Y \rightarrow Y$.

The return time function $\varphi: Y \rightarrow \mathbb{Z}^{+}$satisfies $\int_{Y} \varphi d \mu=\infty$. We require that

$$
\mu(y \in Y: \varphi(y)>n)=\ell(n) n^{-\beta} \text { where } \ell \text { is slowly varying and } \beta \in[0,1] .
$$

Recall that the transfer operator $R: L^{1}(Y) \rightarrow L^{1}(Y)$ for the first return map $F: Y \rightarrow Y$ is defined via the formula $\int_{Y} R v w d \mu=\int_{Y} v w \circ F d \mu, w \in L^{\infty}(Y)$. Let $\mathbb{D}=\{z \in \mathbb{C}:|z|<1\}$ and $\overline{\mathbb{D}}=\{z \in \mathbb{C}:|z| \leq 1\}$. Given $z \in \overline{\mathbb{D}}$, we define $R(z): L^{1}(Y) \rightarrow L^{1}(Y)$ to be the operator $R(z) v=R\left(z^{\varphi} v\right)$. Also, for each $n \geq 1$, we define $R_{n}: L^{1}(Y) \rightarrow L^{1}(Y), R_{n} v=R\left(1_{\{\varphi=n\}} v\right)$. It is easily verified that $R(z)=\sum_{n=1}^{\infty} R_{n} z^{n}$.

Our assumptions on the first return map $F: Y \rightarrow Y$ are functional-analytic. We assume that there is a function space $\mathcal{B} \subset L^{\infty}(Y)$ containing constant functions, with norm \|\| satisfying $|v|_{\infty} \leq\|v\|$ for $v \in \mathcal{B}$, such that

$\left(\mathrm{H} 1^{\prime}\right) R_{n}: \mathcal{B} \rightarrow \mathcal{B}$ are bounded linear operators satisfying $\sum_{n=1}^{\infty}\left\|R_{n}\right\|<\infty$ 
It follows that $z \mapsto R(z)$ is a continuous family of bounded linear operators on $\mathcal{B}$ for $z \in \overline{\mathbb{D}}$. Since $R(1)=R$ and $\mathcal{B}$ contains constant functions, 1 is an eigenvalue of $R(1)$. Throughout, we assume:

(H2) The eigenvalue 1 is simple and isolated in the spectrum of $R(1)$.

In Section 3, we prove that uniform dual ergodicity holds under (H1') and (H2). However, remainders and higher order theory require an improved version of $\left(\mathrm{H}^{\prime}\right)$. For simplicity, we will assume (H1) below, though many results can be obtained under weaker assumptions.

(H1) There is a constant $C>0$ such that $\left\|R_{n}\right\| \leq C \mu(\varphi=n)$ for all $n \geq 1$.

Remark 2.1 In [31], we studied the asymptotics of the iterates $L^{n}$ under conditions (H1) and (H2) together with an aperiodicity assumption (H2(ii) in [31]). Moreover, generally the existence of an asymptotic expression for $L^{n}$ requires $\beta>\frac{1}{2}$. Uniform dual ergodicity deals with the asymptotics of $\sum_{j=1}^{n} L^{j}$, and we prove in this paper that conditions $\left(\mathrm{H}^{\prime}\right)$ and $(\mathrm{H} 2)$ suffice, with no restriction on $\beta$.

Define the bounded linear operators

$$
T_{n}=1_{Y} L^{n} 1_{Y}, \quad n \geq 0, \quad R_{n}=1_{Y} L^{n} 1_{\{\varphi=n\}}=R 1_{\{\varphi=n\}}, \quad n \geq 1 .
$$

(Here, $T_{0}=I$.) The power series

$$
T(z)=\sum_{n=0}^{\infty} T_{n} z^{n}, \quad z \in \mathbb{D}, \quad R(z)=\sum_{n=1}^{\infty} R_{n} z^{n}, \quad z \in \overline{\mathbb{D}},
$$

are analytic on the open unit disk $\mathbb{D}$, and $R(z)$ is continuous on $\overline{\mathbb{D}}$ by $(\mathrm{H} 1)$. We have the usual relation $T_{n}=\sum_{j=1}^{n} T_{n-j} R_{j}$ for $n \geq 1$, and it follows that $T(z)=$ $I+T(z) R(z)$ on $\mathbb{D}$. Hence $T(z)=(I-R(z))^{-1}$ on $\mathbb{D}$.

\subsection{Asymptotics of $T(z)$ on $\mathbb{D}$}

Under an aperiodicity assumption, we obtained in [31] the asymptotics of $T_{n}$ by estimating the Fourier coefficients of $T\left(e^{i \theta}\right)$. The asymptotic expansion of $T\left(e^{i \theta}\right)$ as $\theta \rightarrow 0$ is a key ingredient of the argument in [31.

The corresponding key ingredients for the results in this paper are the asymptotic expansion of $T\left(e^{-u}\right)$ as $u \rightarrow 0^{+}$(u real) for first order uniform dual ergodicity with remainders, and the asymptotic expansion of $T(z)$ as $z \rightarrow 1(z \in \mathbb{D})$ for higher order results.

We recall the first order asymptotics of $T\left(e^{-u}\right)$ from 31. Denote the spectral projection corresponding to the simple eigenvalue 1 for $R$ by $P v=\int_{Y} v d \mu$. 
Proposition 2.2 ([31, Proposition 7.1]) Assume (H1') and (H2). Suppose that $\mu(\varphi>n)=\ell(n) n^{-\beta}$ where $\ell$ is slowly varying and $\beta \in[0,1]$. Define $\tilde{\ell}(n)=$ $\sum_{j=1}^{n} \ell(j) j^{-1}$. Then

$$
T\left(e^{-u}\right) \sim \begin{cases}\tilde{\ell}\left(\frac{1}{u}\right)^{-1} u^{-1} P, & \beta=1, \\ \Gamma(1-\beta)^{-1} \ell\left(\frac{1}{u}\right)^{-1} u^{-\beta} P, & \beta \in[0,1),\end{cases}
$$

as $u \rightarrow 0^{+}$. (Recall that $A(x) \sim c(x) A$ for bounded linear operators $A(x), A: \mathcal{B} \rightarrow \mathcal{B}$ if $\|A(x)-c(x) A\|=o(c(x))$.)

Remark 2.3 [31, Proposition 7.1] does not contain the case $\beta=0$, but the proof in [31] is easily extended to this case.

We now state three results that are proved in Appendix A. The next lemma provides the first order expansion of $T(z)$ for $z \in \mathbb{D}$.

Lemma 2.4 Assume $\left(H 1^{\prime}\right)$ and (H2). Suppose that $\mu(\varphi>n)=\ell(n) n^{-\beta}$ where $\ell$ is slowly varying and $\beta \in(0,1)$. Write $z=e^{-u+i \theta}, u>0$. Then

$$
\Gamma(1-\beta) T(z) \sim \ell(1 /|u-i \theta|)^{-1}(u-i \theta)^{-\beta} P, \text { as } z \rightarrow 1 .
$$

Assuming (H1) and the existence of a good remainder in the tail probabilities $\mu(\varphi>n)$, one can also obtain higher order asymptotics (or asymptotics with remainder) of $T(z)$ (see [31] for higher order expansions of $T\left(e^{i \theta}\right)$ ).

Lemma 2.5 Assume (H1) and (H2) and let $\beta \in(0,1)$. Suppose that $\mu(\varphi>n)=$ $c\left(n^{-\beta}+H(n)\right)$, where $c>0$ and $H(n)=O\left(n^{-2 \beta}\right)$.

If $\beta \in\left(\frac{1}{2}, 1\right)$, set $c_{H}=-\Gamma(1-\beta)^{-1} \int_{0}^{\infty} H_{1}(x) d x$, where $H_{1}(x)=[x]^{-\beta}-x^{-\beta}+$ $H([x])$. Let $k=\max \{j \geq 0:(j+1) \beta-j>0\}$. Then writing $z=e^{-u+i \theta}, u>0$,

$c \Gamma(1-\beta) T(z)=\left((u-i \theta)^{-\beta}+c_{H}(u-i \theta)^{1-2 \beta}+\cdots+c_{H}^{k}(u-i \theta)^{k-(k+1) \beta}\right) P+O(1)$.

If $\beta \in\left(0, \frac{1}{2}\right]$, we assume further that $H(n)=b(n)+c(n)$ where $b(n)$ is monotone with $b(n)=O\left(n^{-2 \beta}\right)$ and $c(n)$ is summable. Then

$$
c \Gamma(1-\beta) T(z)=(u-i \theta)^{-\beta} P+D(z),
$$

where $D(z)=O(1)$ for $\beta<\frac{1}{2}$ and $D(z)=O\left(\log \frac{1}{|u-i \theta|}\right)$ if $\beta=\frac{1}{2}$.

Finally, we state two results about higher order expansions of $T\left(e^{-u}\right)$ that go beyond the situations covered for $T(z)$ above. We recall the following definition introduced by de Haan [19] (see also [9, Chapter 3]):

Definition 2.6 ([19]) A measurable function $f$ on $(0, \infty)$ is in the class $O \Pi_{L}$ for some slowly varying function $L$ if for any $\alpha \geq 0,|f(\alpha x)-f(x)|=O(L(x))$ as $x \rightarrow \infty$. 
For example, if $f(x)=\log ^{p} x, p \in \mathbb{R}$, then $f \in O \Pi_{L}$ with $L=\log ^{p-1} x$.

Lemma 2.7 Assume (H1) and (H2).

(a) Suppose that $\mu(\varphi>n)=c n^{-\beta}+O\left(n^{-\gamma}\right)$ where $c>0,0<\beta<\gamma<1$. Then

$$
c \Gamma(1-\beta) T\left(e^{-u}\right)=\left(u^{-\beta}+O\left(u^{\gamma-2 \beta}\right)\right) P+O(1), u \in[0,1]
$$

(b) Let $\ell, \hat{\ell}$ be slowly varying functions such that $\ell(x) \rightarrow \infty$ as $x \rightarrow \infty$, with $\ell, \hat{\ell}$, $\hat{\ell}^{-1}$ locally bounded on $[0, \infty)$, and such that $\ell \in O \Pi_{\hat{\ell}}$. Suppose that $\mu(\varphi>n)=$ $\ell(n)^{-1}+O\left(\ell(n)^{-2} \hat{\ell}(n)\right)$. Then

$$
T\left(e^{-u}\right)=(\ell(1 / u)+O(\hat{\ell}(1 / u)) P+O(1), u \in[0,1] .
$$

Remark 2.8 The proof of Theorem 1.1 uses Proposition 2.2, and the convergence rates in Theorem 1.3 (and related results) rely on Lemma 2.7. Lemma 2.5 is required for the asymptotic expansion in Theorem 1.4.

Lemma 2.4 is included because it is clearly an interesting result in its own right even though we do not make explicit use of it in this paper. We note that a proof of Theorem 1.1 for $\beta>0$ can be based on Lemma 2.4 via the methods in Section 4 . However, the proof in Section 3.1 is more elegant. Also, under an aperiodicity assumption, it is immediate from Lemma 2.4 that the Fourier coefficients of $T\left(e^{i \theta}\right)$ coincide with the $T_{n}$, bypassing the tedious calculation in [31, Section 4].

\section{Uniform dual ergodicity with remainders}

In this section, we prove uniform dual ergodicity under hypotheses (H1') and (H2). Assuming (H1) and (H2) and imposing further conditions on the tail probabilities, we obtain remainders in the implied convergence.

Recall that $m(n)=\ell(n)$ for $\beta \in[0,1), m(n)=\tilde{\ell}(n)=\sum_{j=1}^{n} \ell(j) j^{-1}$ for $\beta=1$, $D_{\beta}=\Gamma(1-\beta) \Gamma(1+\beta)$ for $\beta \in(0,1)$ and $D_{0}=D_{1}=1$. The first result of this section reads as

Theorem 3.1 Assume $\left(H 1^{\prime}\right)$ and (H2). Suppose $\mu(\phi>n)=\ell(n) n^{-\beta}$, where $\ell$ is slowly varying and $\beta \in[0,1]$. Then

$$
\lim _{n \rightarrow \infty} D_{\beta} m(n) n^{-\beta} \sum_{j=1}^{n} L^{n} v=\int_{Y} v d \mu,
$$

uniformly on $Y$ for all $v \in \mathcal{B}$. 
The proof of Theorem 3.1 is provided in Subsection 3.1; it relies on a version of Karamata's Tauberian Theorem that gives uniform convergence for positive operators. This is the content of Lemma 3.5. In Subsection 3.2, we show that Theorem 3.1 extends to a large class of observables supported on the whole of $X$ and we prove Theorem 1.1.

The proof of Lemma 3.5 combined with arguments used in [28, Theorems 3.1 and 3.2, Chapter VII] allows us to obtain uniform dual ergodic theorems with remainders. Our next result provides an example.

Theorem 3.2 Assume (H1) and (H2). Let $v \in \mathcal{B}$.

(a) If $\mu(\varphi>n)=c n^{-\beta}+O\left(n^{-\gamma}\right)$, where $c>0, \beta \in(0,1)$ and $\gamma>\beta$, then

$$
c D_{\beta} n^{-\beta} \sum_{j=1}^{n} 1_{Y} L^{j} v=\int_{Y} v d \mu+E_{n} v, \quad\left\|E_{n}\right\|=O(1 / \log n) .
$$

(b) Let $\ell, \hat{\ell}$ be slowly varying functions such that $\ell(x) \rightarrow \infty$ as $x \rightarrow \infty$, with $\ell$, $\hat{\ell}, \hat{\ell}^{-1}$ locally bounded on $[0, \infty)$, and such that $\ell \in O \Pi_{\hat{\ell}}$. If $\mu(\varphi>n)=$ $\ell(n)^{-1}+O\left(\ell(n)^{-2} \hat{\ell}(n)\right)$, then

$$
\sum_{j=1}^{n} 1_{Y} L^{j} v=\{\ell(n)+O(\hat{\ell}(n))\} \int_{Y} v d \mu+E_{n} v, \quad\left\|E_{n}\right\|=O(1) .
$$

Theorem 3.2 is proved in Subsection 3.3 .

Proof of Theorem 1.3 Choose $Y$ as in Proposition C.2 so that $\mu(\varphi>n)=$ $c \log ^{-1} n+O\left(\log ^{-2} n\right)$. Take $\mathcal{B}$ to consist of Hölder or BV functions supported on $Y$ as appropriate. In the notation of Theorem 3.2(b), we have $\ell(n)=c^{-1} \log n$ and $\hat{\ell}=1$. It is well-known that hypotheses (H1) and (H2) are satisfied (see for example [31, Section 11]). Hence the result follows from Theorem $3.2(\mathrm{~b})$.

\subsection{A Karamata Theorem for positive operators}

Let $T\left(e^{-u}\right)=\sum_{j=0}^{\infty} T_{j} e^{-u j}, u>0$, where $T_{j}: \mathcal{B} \rightarrow \mathcal{B}$ are uniformly bounded positive operators. Let $P: \mathcal{B} \rightarrow \mathcal{B}$ be a bounded linear operator.

Proposition 3.3 Suppose that $T\left(e^{-u}\right) \sim L(1 / u) u^{-\beta} P$ as $u \rightarrow 0^{+}$where $\beta>0$ and $L$ is slowly varying. Let $q:[0,1] \rightarrow \mathbb{R}$ be a polynomial satisfying $q(0)=0$. Then

$$
\Gamma(1+\beta) \sum_{j=0}^{\infty} T_{j} q\left(e^{-u j}\right) \sim L(1 / u) u^{-\beta} \int_{0}^{\infty} q\left(e^{-t}\right) d t^{\beta} P \quad \text { as } u \rightarrow 0^{+},
$$

where $\sim$ is in the sense of the operator norm for operators on $\mathcal{B}$. 
Proof First note that $\int_{0}^{\infty} e^{-y} d y^{\beta}=\Gamma(1+\beta)$, and so for any $k>0$,

$$
T(k u) \sim L(1 / u)(k u)^{-\beta} P=(\Gamma(1+\beta))^{-1} L(1 / u) u^{-\beta} \int_{0}^{\infty} e^{-k t} d t^{\beta} P .
$$

Write $q(x)=\sum_{k=1}^{m} b_{k} x^{k}$. Then

$$
\begin{aligned}
\sum_{j=0}^{\infty} T_{j} q\left(e^{-u j}\right) & =\sum_{k=1}^{m} b_{k} \sum_{j=0}^{\infty} T_{j} e^{-u k j}=\sum_{k=1}^{m} b_{k} T(k u) \\
& \sim(\Gamma(1+\beta))^{-1} L(1 / u) u^{-\beta} \sum_{k=1}^{m} b_{k} \int_{0}^{\infty} e^{-k t} d t^{\beta} P \\
& =(\Gamma(1+\beta))^{-1} L(1 / u) u^{-\beta} \int_{0}^{\infty} q\left(e^{-t}\right) d t^{\beta} P .
\end{aligned}
$$

Proposition 3.4 Define $g:[0,1] \rightarrow[0,1], g=1_{\left[e^{-1}, 1\right]}$. Let $\epsilon>0$. Then there is a polynomial $q$ with $q(0)=0$ such that $q \geq g$ on $[0,1]$ and

$$
\int_{0}^{1}(q(x)-g(x)) x^{-3 / 2} d x<\epsilon .
$$

Proof We follow the argument starting at the bottom of page 21 in [28]. Choose $\delta>0$ such that $\delta<(2 e)^{-1}$ and $\delta<\left((2 e)^{3 / 2}+4\right)^{-1} \epsilon$. Let $h:[0,1] \rightarrow[0,1]$ be the continuous function that (i) coincides with $g$ except on the interval $J=\left[e^{-1}-\delta, e^{-1}\right]$, and (ii) is linear on the interval $J$. By the Weierstrass approximation theorem, there is a polynomial $R$ that $\delta$-approximates the continuous function $x^{-1} h(x)+\delta$ on $[0,1]$. Then $q(x)=x R(x)$ satisfies $q(0)=0$ and $q(x) \geq h \geq g$. Also $\int_{0}^{1}(h(x)-$ $g(x)) x^{-3 / 2} d x \leq(2 e)^{3 / 2} \delta$ and $\int_{0}^{1}(q(x)-h(x)) x^{-3 / 2} d x \leq 2 \delta \int_{0}^{1} x^{-1 / 2} d x=4 \delta$. The result follows.

Lemma 3.5 Suppose that $T\left(e^{-u}\right) \sim L(1 / u) u^{-\beta} P$ as $u \rightarrow 0^{+}$where $\beta \geq 0$ and $L$ is slowly varying. Then

$$
\lim _{n \rightarrow \infty} \Gamma(1+\beta) L(n)^{-1} n^{-\beta} \sum_{j=0}^{n} T_{j} v=P v,
$$

uniformly on $Y$ for all $v \in \mathcal{B}$.

Proof First, we consider the case $\beta>0$.

Without loss, we may suppose that $v \geq 0$. Define $g=1_{\left[e^{-1}, 1\right]}$, and given $\epsilon>0$ choose $q$ as in Proposition 3.4. By positivity of the operators $T_{j}$ and Proposition 3.3,

$$
\sum_{j=0}^{n} T_{j}=\sum_{j=0}^{\infty} T_{j} g\left(e^{-j / n}\right) \leq \sum_{j=0}^{\infty} T_{j} q\left(e^{-j / n}\right) \sim(\Gamma(1+\beta))^{-1} L(n) n^{\beta} \int_{0}^{\infty} q\left(e^{-t}\right) d t^{\beta} P,
$$


as $n \rightarrow \infty$. More precisely, there exists $h_{0}(n)=o(1)$ as $n \rightarrow \infty$ such that for any $v \in \mathcal{B}, v \geq 0$, and any $y \in Y$,

$$
\sum_{j=0}^{n}\left(T_{j} v\right)(y) \leq(\Gamma(1+\beta))^{-1} L(n) n^{\beta} \int_{0}^{\infty} q\left(e^{-t}\right) d t^{\beta}\left((P v)(y)+h_{0}(n)\|v\|\right) .
$$

But

$$
\begin{aligned}
\int_{0}^{\infty} q\left(e^{-t}\right) d t^{\beta} & =\int_{0}^{\infty} g\left(e^{-t}\right) d t^{\beta}+\int_{0}^{\infty}\left(q\left(e^{-t}\right)-g\left(e^{-t}\right)\right) d t^{\beta} \\
& =1+\int_{0}^{1}(q(x)-g(x)) \beta\left(\log x^{-1}\right)^{\beta-1} x^{-1} d x \\
& \leq 1+C \int_{0}^{1}(q(x)-g(x)) x^{-3 / 2} d x<1+C \epsilon
\end{aligned}
$$

where $C$ is a constant depending only on $\beta$. Hence,

$$
\left(\Gamma(1+\beta) L(n)^{-1} n^{-\beta} \sum_{j=0}^{n}\left(T_{j} v\right)(y) \leq(1+C \epsilon)\left((P v)(y)+h_{0}(n)\|v\|\right) .\right.
$$

The reverse inequality is proved in the same way, and $\epsilon$ is arbitrary completing the proof for $\beta>0$.

Finally, we indicate the differences when $\beta=0$. The conclusion of Proposition 3.3 is replaced by

$$
\sum_{j=0}^{\infty} T_{j} q\left(e^{-u j}\right) \sim L(1 / u) q(1) P \quad \text { as } u \rightarrow 0^{+} .
$$

Let $q(x)=-7 x^{2}+8 x$. Then $q(0)=0, q(1)=1$, and $q \geq g$ on $[0,1]$. Hence

$$
\sum_{j=0}^{n} T_{j}=\sum_{j=0}^{\infty} T_{j} g\left(e^{-j / n}\right) \leq \sum_{j=0}^{\infty} T_{j} q\left(e^{-j / n}\right) \sim L(n) q(1) P=L(n) P .
$$

For the reverse direction, take $q(x)=2 x^{2}-x$ so that $q(0)=0, q(1)=1$, and $q \leq g$ on $[0,1]$.

Proof of Theorem 3.1 This follows immediately from Proposition 2.2 and Lemma 3.5

\subsection{Uniform dual ergodic theorems for general observables}

Theorem 3.1 establishes uniform dual ergodicity for observables $v \in \mathcal{B}$. In particular, it is required that $v$ is supported on $Y$. However, it is straightforward to generalise [31, Theorem 10.2], and thereby prove uniform dual ergodicity for a large class of observables supported on the whole of $X$. 
Let $X_{k}=f^{-k} Y \backslash \cup_{j=0}^{k-1} f^{-j} Y$. Thus, $z \in X_{k}$ if and only if $k \geq 0$ is the smallest $k$ such that $f^{k} z \in Y$ (In particular, $X_{0}=Y$.). Given $v \in L^{\infty}(X)$, define $v_{k}=1_{X_{k}} v$. Let $\mathcal{B}(X)$ consist of functions $v \in L^{1}(X)$ satisfying $L^{k} v_{k} \in \mathcal{B}$ for each $k \geq 0$.

Theorem 3.6 Assume the set up of Theorem 3.1. Let $v \in \mathcal{B}(X)$ and suppose that $\sum\left\|L^{k} v_{k}\right\|<\infty$. Then

$$
\lim _{n \rightarrow \infty} D_{\beta} m(n) n^{-\beta} \sum_{j=1}^{n} L^{n} v=\int_{X} v d \mu, \quad \text { uniformly on } Y .
$$

Proof Set $a_{n}=D_{\beta}^{-1} m(n)^{-1} n^{\beta}$. By Theorem 3.1, $\sum_{j=0}^{n} T_{j}=a_{n} P+D_{n}$, where $\left\|D_{n}\right\|=o\left(a_{n}\right)$. On $Y$,

$$
\sum_{j=0}^{n} L^{j} v=\sum_{j=0}^{n} \sum_{\ell=0}^{j} T_{j-\ell} L^{\ell} v_{\ell}=\sum_{\ell=0}^{n} \sum_{j=0}^{n-\ell} T_{j} L^{\ell} v_{\ell} .
$$

Thus,

$$
\begin{array}{r}
\left\|a_{n}^{-1} \sum_{j=0}^{n} L^{j} v-\int_{X} v d \mu\right\| \ll\left\|a_{n}^{-1} \sum_{\ell=0}^{n} a_{n-\ell} \int_{X} L^{\ell} v_{\ell} d \mu-\sum_{\ell=0}^{n} \int_{X} v_{\ell} d \mu\right\| \\
+a_{n}^{-1} \sum_{\ell=0}^{n}\left\|D_{n-\ell}\right\|\left\|L^{\ell} v_{\ell}\right\|+\sum_{\ell>n} \int_{X}\left|v_{\ell}\right| d \mu \\
=\sum_{\ell=0}^{n}\left(\frac{a_{n-\ell}}{a_{n}}-1\right) \int_{X} v_{\ell} d \mu+\sum_{\ell=0}^{n} a_{n}^{-1}\left\|D_{n-\ell}\right\|\left\|L^{\ell} v_{\ell}\right\|+\sum_{\ell>n} \int_{X}\left|v_{\ell}\right| d \mu .
\end{array}
$$

Clearly, the last term converges to zero. Moreover, $\lim _{n \rightarrow \infty}\left(a_{n}^{-1} a_{n-\ell}-1\right)=0$, for each $\ell$. Since we also know that $\int_{X} v_{\ell} d \mu$ is summable, the first term converges to zero. Finally, $\lim _{n \rightarrow \infty} a_{n}^{-1}\left\|D_{n-\ell}\right\|=0$, for each $\ell$. This together with the summability of $\left\|L^{\ell} v_{\ell}\right\|$ shows that the second term converges to zero, ending the proof.

Proof of Theorem 1.1 We modify the proof of [31, Theorem 11.5]. Without loss, choose $Y$ such that $f(Y)=X$. Suppose first that $v: X \rightarrow \mathbb{R}$ is $\mu$-integrable and BV. As noted in [31, Section 11.3], the space $\mathcal{B}=B V(Y)$ is a suitable Banach space. In particular, hypotheses (H1) and (H2) are satisfied and moreover $\sum\left\|L^{k} v_{k}\right\|<\infty$. Hence the hypotheses of Theorem 3.6 are satisfied and we obtain a uniform dual ergodic theorem for such $v$. Finally, given $v$ of the required form $v=\xi u$, we approximate $u$ from above and below by BV functions $u^{ \pm}$. Then $v$ is approximated from above and below by observables $v^{ \pm}=\xi u^{ \pm}$for which uniform convergence on compact sets of $X^{\prime}$ holds. But $\int\left(v^{+}-v^{-}\right)$can be made arbitrarily small and the result follows. 


\subsection{Remainders in the uniform convergence}

The proof of Theorem 3.2 relies on the following Tauberian remainder theorem for positive operators. Let $T\left(e^{-u}\right), P$ be bounded linear operators on $\mathcal{B}, u \in(0,1)$.

Lemma 3.7 (a) Let $\beta>0, \hat{\beta}<\beta$. Suppose that $T\left(e^{-u}\right)=u^{-\beta} P+D(u)$ as $u \rightarrow 0^{+}$, where $\|D(u)\|=O\left(u^{-\hat{\beta}}\right)$. Then

$$
\Gamma(1+\beta) \sum_{j=0}^{n} T_{j}=n^{\beta} P+E_{n}, \quad\left\|E_{n}\right\|=O\left(n^{\beta} / \log n\right) .
$$

(b) Let $\ell, \hat{\ell}$ be slowly varying functions such that $\ell(x) \rightarrow \infty$ as $x \rightarrow \infty$ and $\ell \in O \Pi_{\hat{\ell}}$. Suppose that $T\left(e^{-u}\right)=\{\ell(1 / u)+O(\hat{\ell}(1 / u))\} P+D(u)$ as $u \rightarrow 0^{+}$, where $\|D(u)\|=$ $O(1)$. Then

$$
\sum_{j=0}^{n} T_{j}=\left(\ell(n)+O(\hat{\ell}(1 / n)) P+E_{n}, \quad\left\|E_{n}\right\|=O(1) .\right.
$$

Proof We mimic the arguments used in the proof of [28, Theorem 3.1, Chapter VII]. Define $g:[0,1] \rightarrow[0,1], g=1_{\left[e^{-1}, 1\right]}$. By [28, Theorem 3.4, Chapter VII] (see also [26, 27]), there exist constants $m_{0} \geq 1, C_{1}>0, C_{2}>1$, and for every $m \geq m_{0}$ there exist real polynomials $q_{m}^{j}(x)=\sum_{k=1}^{m} b_{k m}^{j} x^{k}, j=1,2$, such that

(i) $q_{m}^{1}(x) \leq g(x) \leq q_{m}^{2}(x), 0 \leq x \leq 1$.

(ii) $\int_{0}^{\infty}\left(q_{m}^{2}(x)-q_{m}^{1}(x)\right) d t^{\beta} \leq C_{1} / m$.

(iii) $\sum_{k=1}^{m}\left|b_{k m}^{j}\right| \leq C_{2}^{m}$ for $j=1,2$.

Proceeding as in the proof of Lemma 3.5, in case $(a)$, we have

$$
\begin{aligned}
\sum_{j=0}^{n} T_{j} & =\sum_{j=0}^{\infty} T_{j} g\left(e^{-j / n}\right) \leq \sum_{j=0}^{\infty} T_{j} q_{m}^{2}\left(e^{-j / n}\right)=\sum_{k=1}^{m} b_{k m}^{2} T(k / n) \\
& =n^{\beta} \sum_{k=1}^{m} b_{k m}^{2} k^{-\beta} P+\sum_{k=1}^{m} b_{k m}^{2} D(k / n) .
\end{aligned}
$$

Moreover,

$$
\begin{aligned}
\Gamma(1+\beta) \sum_{k=1}^{m} b_{k m}^{2} k^{-\beta} & =\sum_{k=1}^{m} b_{k m}^{2} \int_{0}^{\infty} e^{-k t} d t^{\beta}=\int_{0}^{\infty} q_{m}^{2}\left(e^{-t}\right) d t^{\beta} \\
& =1+\int_{0}^{\infty}\left(q_{m}^{2}\left(e^{-t}\right)-g\left(e^{-t}\right)\right) d t^{\beta}
\end{aligned}
$$


Hence

$\Gamma(1+\beta) n^{-\beta} \sum_{j=0}^{n} T_{j} \leq P+\int_{0}^{\infty}\left(q_{m}^{2}\left(e^{-t}\right)-g\left(e^{-t}\right)\right) d t^{\beta} P+\Gamma(1+\beta) n^{-\beta} \sum_{k=1}^{m} b_{k m}^{2} D(k / m)$.

By property (i) and (ii) above, $\int_{0}^{\infty}\left(q_{m}^{2}\left(e^{-t}\right)-g\left(e^{-t}\right)\right) d t^{\beta} \leq C_{1} / m$. By property (iii) and the hypothesis on $D(u)$,

$$
\left\|\sum_{k=1}^{m} b_{k m}^{2} D(k / m)\right\| \ll n^{\gamma} \sum_{k=1}^{m} k^{-\gamma}\left|b_{k m}^{2}\right| \leq n^{\gamma} \sum_{k=1}^{m}\left|b_{k m}^{2}\right| \leq C_{2}^{m} n^{\gamma} .
$$

Hence, taking $m=\delta \log n$ with $\delta$ sufficiently small, we have $\Gamma(1+\beta) n^{-\beta} \sum_{j=0}^{n} T_{j}-P \leq$ $H_{n}$ where $\left\|H_{n}\right\| \ll 1 / \log n$. Repeating the argument with $q_{m}^{1}$ instead of $q_{m}^{2}$, we obtain the inequality in the reverse direction.

In case $(b)$, we use two fixed polynomials $q^{j}(x)=\sum_{k=1}^{m} b_{k}^{j} x^{k}, j=1,2$ as in the proof of Lemma 3.5, so that $q^{1} \leq g \leq q^{2}$ in $[0,1]$ and $q^{j}(0)=0, q^{j}(1)=1$. Write $T\left(e^{-u}\right)=\{\ell(1 / u)+h(u)\} P+D(u)$ as $u \rightarrow 0^{+}$, where $h(u)=O(\hat{\ell}(1 / u))$. Then

$$
\begin{aligned}
\sum_{j=0}^{n} T_{j} & \leq \sum_{k=1}^{m} b_{k}^{2} \ell(n / k) P+\sum_{k=1}^{m} b_{k}^{2} h(k / n) P+\sum_{k=1}^{m} b_{k}^{2} D(k / n) \\
& =\ell(n) \sum_{k=1}^{m} b_{k}^{2} P+\sum_{k=1}^{m} b_{k}^{2}(\ell(n / k)-\ell(n)) P+\sum_{k=1}^{m} b_{k}^{2} h(k / n) P+H_{n} \\
& =\ell(n) P+O(\hat{\ell}(n)) P+H_{n},
\end{aligned}
$$

where $\left\|H_{n}\right\|=O(1)$. The reverse inequality is obtained by repeating the argument with $q^{1}$ instead of $q^{2}$.

Proof of Theorem 3.2 This is immediate from Lemma 2.7 and Lemma 3.7.

Remark 3.8 As mentioned in the introduction, it seems unlikely that the apparently weak estimate in Theorem 3.2(a) can be improved using methods from real Tauberian theory. We explain this now within the context of the classical scalar theory.

The method of approximation by polynomials links information about the asymptotics of real power series $\Phi(s)=\sum_{n=0}^{\infty} u_{n} e^{-n s}$ as $s \rightarrow 0^{+}$, to asymptotics of the partial sums $\sum_{j=0}^{n} u_{j}$ as $n \rightarrow \infty$ (see Korevaar [28, Chapters I and VII]). Freud [13] refined Karamata's method and obtained error estimates of the type described in Theorem 3.2 (a). Within the context of real power series, the Freud remainder terms are optimal. For example, suppose that the coefficients $u_{n}$ are non-negative and $\Phi(s)=s^{-\beta}+O\left(s^{-\gamma}\right)$ as $s \rightarrow 0^{+}$, where $\beta>0$ and $\gamma<\beta$. By Ingham [22] and Korevaar [26, 27], the best possible result for the asymptotics of the partial sums is $\sum_{j=0}^{n} u_{j}=n^{\beta}+O\left(n^{\beta} / \log n\right)$ as $n \rightarrow \infty$. 
More generally, Tauberian theorems relate the asymptotics of a nondecreasing function $U(x)$ as $x \rightarrow \infty$ with the asymptotics of the Laplace transform $\hat{U}(s)=$ $\int_{0}^{\infty} e^{-s x} d U(x)$ as $s \rightarrow 0^{+}$. Improved remainder theorems can be obtained by imposing further conditions on $U(x)$ (see for instance [7, 17, 18, 20, 23, 33]; see also [9, Chapter 3] and references therein). However, such conditions on $U$ are not appropriate in the context of dynamical systems.

\section{Higher order asymptotics}

In this section, we prove a result on higher order asymptotics in the uniform dual ergodic theorem. Throughout, we assume (H1) and (H2), and that $\mu(\varphi>n)=$ $c\left(n^{-\beta}+H(n)\right)$, where $\beta \in(0,1), c>0$ and $H(n)=O\left(n^{-2 \beta}\right)$. If $\beta \in\left(0, \frac{1}{2}\right]$, we assume further that $H(n)=b(n)+c(n)$ where $b(n)$ is monotone with $b(n)=O\left(n^{-2 \beta}\right)$ and $c(n)$ is summable.

Recall $H_{1}(x)=[x]^{-\beta}-x^{-\beta}+H([x])$. For $\beta \in\left(\frac{1}{2}, 1\right), c_{H}=-\Gamma(1-\beta)^{-1} \int_{0}^{\infty} H_{1}(x) d x$ and we set $d_{k}=c_{H}^{k} / \Gamma((k+1) \beta-(k-1))$ for $k \geq 0$.

Theorem 4.1 Let $k=\max \{j \geq 0:(j+1) \beta-j>0\}$. Then for any $\epsilon>0$,

$$
c \Gamma(1-\beta) \sum_{j=0}^{n-1} T_{j}=\left(d_{0} n^{\beta}+d_{1} n^{2 \beta-1}+d_{2} n^{3 \beta-2}+\cdots+d_{k} n^{(k+1) \beta-k}\right) P+O\left(n^{\epsilon}\right) .
$$

Proof of Theorem 1.4 Choose $Y$ as in Proposition C.1. Take $\mathcal{B}$ to consist of Hölder or BV functions supported on $Y$ as appropriate. Again, it is well-known that hypotheses (H1) and (H2) are satisfied [31, Section 11]. Hence the result follows from Theorem 4.1 and Proposition C.1.

In the remainder of this section, we prove Theorem 4.1. The following result can be found in Korevaar [28, Proposition 16.1, Chapter III], see also [36].

Lemma 4.2 Let $p \in \mathbb{N}$ fixed and let $0<r<1$. Then for all $\alpha \in(0,1]$ with $1-r \leq \alpha / 4$ and for $m \in \mathbb{Z}$, the following holds:

$$
\begin{aligned}
& \int_{-\alpha}^{\alpha} \frac{\left(e^{i \theta}-e^{i \alpha}\right)^{p}\left(e^{i \theta}-e^{-i \alpha}\right)^{p}}{1-r e^{i \theta}} e^{i m \theta} d \theta \\
& = \begin{cases}O\left(\frac{\alpha^{2 p}}{\alpha^{p}|m|^{p}+1}\right), & m \geq-(2 p-1) \\
2 \pi r^{-(2 p+m)}\left(1-2 r \cos \alpha+r^{2}\right)^{p}+O\left(\frac{\alpha^{2 p}}{\alpha^{p}|m|^{p}+1}\right), & m \leq-2 p\end{cases}
\end{aligned}
$$

where the implied constants are independent of $r, \alpha, m$.

Recall $T(z)=\sum_{j=0}^{\infty} T_{j} z^{j}$ for $|z|<1$. An immediate consequence of the above proposition is 
Corollary 4.3 (cf $[28$, Corollary 16.2, Chaper III $]$ ) Let $p \in \mathbb{N}$ be fixed. Then for all $r \in(0,1), \alpha \in(0,1]$ with $1-r \leq \alpha / 4$, and $n \geq 2 p$,

$2 \pi r^{n-2 p}\left(1-2 r \cos \alpha+r^{2}\right)^{p} \sum_{j=0}^{n-2 p} T_{j}=\int_{-\alpha}^{\alpha} \frac{T\left(r e^{i \theta}\right)}{1-r e^{i \theta}}\left(e^{i \theta}-e^{i \alpha}\right)^{p}\left(e^{i \theta}-e^{-i \alpha}\right)^{p} e^{-i n \theta} d \theta+B$, where $\|B\|=O\left(\sum_{j=0}^{\infty} \frac{\left\|T_{j}\right\| r^{j} \alpha^{2 p}}{\alpha^{p}|j-n|^{p}+1}\right)$.

Proof This is exactly the same as the proof of [28, Corollary 16.2, Chaper III] formulated for the scalar case.

Below we collect some useful tools for the proof of Theorem 4.1 .

Proposition 4.4 Let $\theta \in[-\pi / 2, \pi / 2]$. Then $\left|1-e^{-1 / n} e^{i \theta}\right|^{-1} \ll n$ and $\mid 1-$ $\left.e^{-1 / n} e^{i \theta}\right|^{-1} \ll 1 /|\theta|$.

Proof To obtain the first estimate, we use the fact that $\left|1-e^{-1 / n} e^{i \theta}\right| \geq \operatorname{Re}(1-$ $\left.e^{-1 / n} e^{i \theta}\right) \geq 1-e^{-1 / n}$. Hence, $\left|1-e^{-1 / n} e^{i \theta}\right|^{-1} \ll n$.

To obtain the second estimate, we use the fact that $\left|1-e^{-1 / n} e^{i \theta}\right| \geq \mid \operatorname{Im}(1-$ $\left.e^{-1 / n} e^{i \theta}\right)|=| \sin \theta|\gg| \theta \mid$. Hence, $\left|1-e^{-1 / n} e^{i \theta}\right|^{-1} \ll 1 /|\theta|$.

Proposition 4.5 Let $\gamma \in(0,1 / 2)$ fixed and let $A(n):=1-2 e^{-1 / n} \cos \left(1 / n^{\gamma}\right)+e^{-2 / n}$. Let $A(\theta, n):=1-2 e^{i \theta} \cos \left(1 / n^{\gamma}\right)+e^{2 i \theta}$ for $\theta \in\left[-1 / n^{\gamma}, 1, n^{\gamma}\right]$. Then the following hold:

(a) $\left|A^{-1}(n)\right| \ll n^{2 \gamma}$ and $\left|A^{-1}(n) A(\theta, n)\right|=O(1)$, uniformly in $\theta \in\left[-1 / n^{\gamma}, 1 / n^{\gamma}\right]$.

(b) $\left|A^{-2}(n)\left(A^{2}(\theta, n)-A^{2}(n)\right)\right|=O(1 / n)$, uniformly in $\theta \in[-1 / n, 1 / n]$.

(c) If $\theta \in\left[-1 / n^{\gamma},-1 / n\right] \cup\left[1 / n, 1 / n^{\gamma}\right]$ then $\left|A^{-2}(n)\left(A^{2}(\theta, n)-A^{2}(n)\right)\right| \ll n^{2 \gamma} \theta^{2 \gamma+1}$.

Proof (a) Write $A(n)=\left(1-e^{-1 / n} e^{i / n^{\gamma}}\right)\left(1-e^{-1 / n} e^{-i / n^{\gamma}}\right)$ and $A(\theta, n)=\left(e^{i \theta}-\right.$ $\left.e^{i / n^{\gamma}}\right)\left(e^{i \theta}-e^{-i / n^{\gamma}}\right)$. Setting $\theta= \pm 1 / n^{\gamma}$ in Proposition 4.4 yields the estimate for $A^{-1}(n)$. Clearly, $|A(\theta, n)| \ll 1 / n^{2 \gamma}$ for $\theta \in\left[-1 / n^{\gamma}, 1, n^{\gamma}\right]$, yielding the second estimate.

(b) Let $A_{1}(\theta, n):=A(\theta, n)-A(n)=\left(e^{i \theta}-e^{-1 / n}\right)\left(e^{i \theta}+e^{-1 / n}-2 \cos \left(1 / n^{\gamma}\right)\right)$. Since $\gamma<1 / 2,\left|A_{1}(\theta, n)\right| \ll(|\theta|+1 / n)\left(|\theta|+1 / n^{2 \gamma}\right) \ll n^{-2 \gamma-1}$ uniformly in $\theta \in$ $[-1 / n, 1 / n]$. Recall that $\left|A^{-1}(n)\right| \ll n^{2 \gamma}$ and that $\left|A^{-1}(n) A(\theta, n)\right|=O(1)$. Writing $A^{2}(\theta, n)-A^{2}(n)=(A(\theta, n)+A(n)) A_{1}(\theta, n)$, we see that

$$
\left|A^{-2}(n)\left(A^{2}(\theta, n)-A^{2}(n)\right)\right| \ll\left|A^{-1}(n) A_{1}(\theta, n)\right| \ll n^{2 \gamma} n^{-(2 \gamma+1)}=1 / n .
$$

(c) If $\theta \in\left[-1 / n^{\gamma},-1 / n\right] \cup\left[1 / n, 1 / n^{\gamma}\right]$, then $\left|A_{1}(\theta, n)\right| \ll|\theta|\left(|\theta|+1 / n^{2 \gamma}\right) \ll|\theta|^{2 \gamma+1}$. Thus, $\left|A^{-2}(n)\left(A^{2}(\theta, n)-A^{2}(n)\right)\right| \ll n^{2 \gamma}|\theta|^{2 \gamma+1}$. 
Proof of Theorem 4.1 We work out the implications of Corollary 4.3, Fix $p=2$, $\gamma \in(0, \beta) \cap\left(0, \frac{1}{2}\right)$ and let $r=e^{-1 / n}, \alpha=n^{-\gamma}$ (in particular, the constraint $1-r \leq \alpha / 4$ is satisfied) and write

$$
\frac{2 \pi}{e} e^{4 / n} A^{2}(n) \sum_{j=0}^{n-4} T_{j}=\int_{-\alpha}^{\alpha} \frac{T\left(e^{-1 / n} e^{i \theta}\right)}{1-e^{-1 / n} e^{i \theta}} A^{2}(\theta, n) e^{-i n \theta} d \theta+B
$$

where

$$
\|B\|=O\left(\sum_{j=0}^{\infty} \frac{\left\|T_{j}\right\| \alpha^{4}}{\alpha^{2}(j-n)^{2}+1}\right)
$$

By Lemma 2.4, $\left\|T\left(e^{-1 / n} e^{i \theta}\right)\right\| \ll\left|\frac{1}{n}-i \theta\right|^{-\beta} \leq|\theta|^{-\beta}$. Hence, for all $j \geq 0$,

$$
|| T_{j}|| \ll \int_{-\pi}^{\pi}|\theta|^{-\beta} d \theta=O(1) .
$$

Thus,

$$
\|B\| \ll \alpha^{4}\left(\sum_{|j-n| \leq 1 / \alpha} \frac{1}{\alpha^{2}(j-n)^{2}+1}+\sum_{|j-n| \geq 1 / \alpha} \frac{1}{\alpha^{2}(j-n)^{2}+1}\right) \ll \alpha^{4}(1 / \alpha)=n^{-3 \gamma} .
$$

By Proposition 4.5(a), $A^{-2}(n) \ll n^{4 \gamma}$. Multiplying (4.2) by $A^{-2}(n)$, we have

$$
\frac{2 \pi}{e} e^{4 / n} \sum_{j=0}^{n-4} T_{j}=\int_{-n^{-\gamma}}^{n^{-\gamma}} \frac{T\left(e^{-1 / n} e^{i \theta}\right)}{\left(1-e^{-1 / n} e^{i \theta}\right)} \frac{A^{2}(\theta, n)}{A^{2}(n)} e^{-i n \theta} d \theta+O\left(n^{\gamma}\right)=I+O\left(n^{\gamma}\right) .
$$

Next, write

$I=\int_{-1 / n^{\gamma}}^{1 / n^{\gamma}} \frac{T\left(e^{-1 / n} e^{i \theta}\right)}{1-e^{-1 / n} e^{i \theta}} e^{-i n \theta} d \theta+\int_{-1 / n^{\gamma}}^{1 / n^{\gamma}} \frac{T\left(e^{-1 / n} e^{i \theta}\right)}{1-e^{-1 / n} e^{i \theta}} \frac{A^{2}(\theta, n)-A^{2}(n)}{A^{2}(n)} e^{-i n \theta} d \theta=J+H$

We first estimate $H$. Put $F(\theta, n):=T\left(e^{-1 / n} e^{i \theta}\right)\left(1-e^{-1 / n} e^{i \theta}\right)^{-1} A^{-2}(n)\left(A^{2}(\theta, n)-\right.$ $\left.A^{2}(n)\right)$. Recall $\left\|T\left(e^{-1 / n} e^{i \theta}\right)\right\| \ll\left|\frac{1}{n}-i \theta\right|^{-\beta} \ll n^{\beta}$. By Proposition 4.4, $\left|1-e^{-1 / n} e^{i \theta}\right|^{-1} \ll n$. This together with Proposition 4.5(b) implies that $\left\|1_{[-1 / n, 1 / n]} F(\theta, n)\right\| \ll n^{\beta}$. Thus, $\left\|\int_{-1 / n}^{1 / n} F(\theta, n) d \theta\right\| \ll n^{\beta-1}$.

By Proposition 4.4, $\left|1-e^{-1 / n} e^{i \theta}\right|^{-1} \ll 1 /|\theta|$. Hence, $\| T\left(e^{-1 / n} e^{i \theta}\right)(1-$ $\left.e^{-1 / n} e^{i \theta}\right)^{-1} \| \ll|\theta|^{-(\beta+1)}$. This together with Proposition 4.5)(c) implies that $\left\|1_{a} F(\theta, n)\right\| \ll n^{2 \gamma}|\theta|^{2 \gamma-\beta}$, where $a:=\left[-1 / n^{\gamma},-1 / n\right] \cup\left[1 / n, 1 / n^{\gamma}\right]$. Thus, $\left\|\int_{a} F(\theta, n) d \theta\right\| \ll n^{2 \gamma} \int_{a}|\theta|^{2 \gamma-\beta} d \theta \ll n^{\gamma \beta+\gamma-2 \gamma^{2}}$. Putting the two estimates together, we have $\|H\| \ll n^{\gamma(\beta+1-2 \gamma)}$.

Next, we estimate $J$. Since $\left(1-e^{-1 / n} e^{i \theta}\right)^{-1}=\left(\frac{1}{n}-i \theta\right)^{-1}\left(1+O\left(\left|\frac{1}{n}-i \theta\right|\right)\right)$,

$$
J=\int_{-1 / n^{\gamma}}^{1 / n^{\gamma}} \frac{T\left(e^{-1 / n} e^{i \theta}\right)}{\frac{1}{n}-i \theta} e^{-i n \theta} d \theta+\int_{-1 / n^{\gamma}}^{1 / n^{\gamma}} T\left(e^{-1 / n} e^{i \theta}\right) h(\theta, n) d \theta
$$


where $|h(\theta, n)|=O(1)$. But $\left\|\int_{-1 / n^{\gamma}}^{1 / n^{\gamma}} T\left(e^{-1 / n} e^{i \theta}\right) h(\theta, n) d \theta\right\| \ll \int_{-1 / n^{\gamma}}^{1 / n^{\gamma}}|\theta|^{-\beta} d \theta \ll$ $n^{-\gamma(1-\beta)}$, so $J=J_{1}+O\left(n^{-\gamma(1-\beta)}\right)$ where $J_{1}=\int_{-1 / n^{\gamma}}^{1 / n^{\gamma}} \frac{T\left(e^{-1 / n} e^{i \theta}\right)}{\frac{1}{n}-i \theta} e^{-i n \theta} d \theta$. By Lemma 2.5,

$$
c \Gamma(1-\beta) T(z)=\sum_{j=0}^{k} c_{H}^{j}\left(\frac{1}{n}-i \theta\right)^{j-(j+1) \beta} P+D(z)
$$

where in the worst case $\left(\beta=\frac{1}{2}\right),\|D(z)\|=O\left(\log \frac{1}{|u-i \theta|}\right)$. Thus, we can write

$$
c \Gamma(1-\beta) J_{1}=\sum_{j=0}^{k} c_{H}^{j} L_{j} P+J^{\prime}
$$

where

$$
L_{j}=\int_{-1 / n^{\gamma}}^{1 / n^{\gamma}} \frac{e^{-i n \theta}}{\left(\frac{1}{n}-i \theta\right)^{(j+1) \beta-(j-1)}} d \theta
$$

and

$$
\left\|J^{\prime}\right\| \ll \int_{-1 / n^{\gamma}}^{1 / n^{\gamma}} \frac{\log \frac{1}{\left|\frac{1}{n}-i \theta\right|}}{\left|\frac{1}{n}-i \theta\right|} d \theta \ll \log n \int_{0}^{1 / n} n d \theta+\log n \int_{1 / n}^{1 / n^{\gamma}} \frac{1}{\theta} d \theta \ll(\log n)^{2} .
$$

By Corollary B.3 (with $\rho=(j+1) \beta-j)$,

$$
L_{j}=\frac{2 \pi}{e} \frac{n^{(j+1) \beta-j}}{\Gamma((j+1) \beta-(j-1))}+O\left(n^{\gamma((j+1) \beta)-j)}\right) .
$$

Putting all the estimates together,

$$
c \Gamma(1-\beta) I=\frac{2 \pi}{e}\left(\sum_{j=0}^{k} d_{j} n^{(j+1) \beta-j}\right) P+O\left(n^{\gamma(\beta+1-2 \gamma)}\right) .
$$

The conclusion follows by plugging this estimate into (4.4) and taking $\gamma$ sufficiently small.

Remark 4.6 The proof of Theorem 1.7 goes exactly same as the proof of Theorem 4.1 with $T(z)$ replaced by $\Phi(z), \sum_{j=0}^{n-1} T_{j}$ replaced by $\sum_{j=0}^{n-1} u_{j}$ and $j-(j+1) \beta$ replaced by $\gamma_{j}, j=1, \ldots, k$. The asymptotics of $\Phi(z)$ is part of the hypothesis of Theorem 1.7 .

\section{A Proof of Lemmas 2.4, 2.5 and 2.7}

By $\left(\mathrm{H}^{\prime}\right)$ and $(\mathrm{H} 2)$, there exists $\epsilon>0$ and a continuous family of simple eigenvalues

of $R(z)$, namely $\lambda(z)$ for $z \in \overline{\mathbb{D}} \cap B_{\epsilon}(1)$ with $\lambda(1)=1$. Let $P(z): \mathcal{B} \rightarrow \mathcal{B}$ denote the corresponding family of spectral projections with $P(1)=P$ and complementary 
projections $Q(z)=I-P(z)$. Also, let $v(z) \in \mathcal{B}$ denote the corresponding family of eigenfunctions normalized so that $\int_{Y} v(z) d \mu=1$ for all $z$. In particular, $v(1) \equiv 1$.

Then we can write

$$
T(z)=(1-\lambda(z))^{-1} P(z)+(I-R(z))^{-1} Q(z)
$$

for $z \in \overline{\mathbb{D}} \cap B_{\epsilon}(1), z \neq 1$.

Proposition A.1 Assume $\left(H 1^{\prime}\right)$ and (H2). There exists $\epsilon, C>0$ such that $\|(I-$ $R(z))^{-1} Q(z) \| \leq C$ for $z \in \overline{\mathbb{D}} \cap B_{\epsilon}(1), z \neq 1$.

Proof of Lemma 2.4 The main part of the proof is to show that $1-\lambda(z) \sim$ $\Gamma(1-\beta) \ell(1 /|u-i \theta|)(u-i \theta)^{\beta}$. By (A.1), continuity of $P(z)$, and Proposition A.1, $T(z)=(1-\lambda(z))^{-1}(P+o(1))+O(1)$ and the result follows.

For the asymptotics of $1-\lambda(z)$, we follow [4, 31]. Note that $|v(z)-1|_{\infty}=o(1)$ as $z \rightarrow 1$. Define the distribution function $G(x)=\mu(\varphi \leq x)$. Choose $\epsilon>0$ such that $\lambda(z)$ is well defined for $z=e^{-u+i \theta}$ for $u>0$ and $\theta \in(-\epsilon, \epsilon)$. Then $\lambda(z) v(z)=$ $R(z) v(z)=R\left(e^{(-u+i \theta) \varphi} v(z)\right)$, and so $\lambda(z)=\int_{Y} \lambda(z) v(z) d \mu=\int_{Y} e^{(-u+i \theta) \varphi} v(z) d \mu=$ $1-\int_{Y}\left(1-e^{(-u+i \theta) \varphi}\right) v(z) d \mu$.

Let $\mathcal{F}_{0}$ denote the $\sigma$-algebra generated by $\varphi$, namely the partition into the sets $\{\varphi=n\}$. Define the step function $\hat{v}(z):[0, \infty) \rightarrow \mathbb{C}$ given by $\hat{v}(z) \circ \varphi=E\left(v(z) \mid \mathcal{F}_{0}\right)$. Then $\lambda(z)=1-\int_{0}^{\infty}\left(1-e^{(-u+i \theta) x)}\right) \hat{v}_{z}(x) d G(x)$, where $\left|\hat{v}_{z}(x)-1\right|=o(1)$, uniformly in $x$, as $z \rightarrow 1$.

Define $\hat{G}_{z}$ such that $d \hat{G}_{z}=\hat{v}_{z} d G$. Integrating by parts,

$$
1-\lambda(z)=\int_{0}^{\infty}\left(1-e^{(-u+i \theta) x)}\right) d \hat{G}_{z}(x)=(u-i \theta) \int_{0}^{\infty} e^{-(u-i \theta) x} g_{z}(x)(1-G(x)) d x
$$

where $\left|g_{z}(x)-1\right|=o(1)$, uniformly in $x$, as $z \rightarrow 1$.

Since $1-G(x)=x^{-\beta} \ell(x)$, we can write $1-\lambda(z)=\ell(1 /|u-i \theta|)(u-i \theta)^{\beta} J(z)$, where

$$
J(z)=\int_{0}^{\infty} \frac{e^{-(u-i \theta) x}}{[(u-i \theta) x]^{\beta}} h_{u, \theta}(x) g_{u, \theta}(x)(u-i \theta) d x, \quad h_{u, \theta}(x)=\frac{\ell(x)}{\ell(1 /|u-i \theta|)} .
$$

Here, $\left|g_{u, \theta}-1\right|_{\infty}=o(1)$ as $u, \theta \rightarrow 0$. The proof will be complete once we show that $J(z)$ converges to $\Gamma(1-\beta)$ as $u, \theta \rightarrow 0$. We consider only the case $\theta>0$, since the case $\theta<0$ follows by the same argument.

We consider separately each of two possible cases: (i) $0 \leq \theta \leq u$; (ii) $0 \leq u \leq \theta$. In both cases we let $\hat{h}_{u, \theta}(\sigma)=h_{u, \theta}(\sigma /|u-i \theta|)$.

In case $(i)$ we let $I_{1}=\int_{0}^{\infty} e^{-(u-i \theta) x}[(u-i \theta) x]^{-\beta}(u-i \theta) d x$. Put $y=\theta / u$. Using 
the substitution $\sigma=|u-i \theta| x$, we have

$$
\begin{aligned}
J(z)=I_{1} & +\left(\frac{1-i y}{|1-i y|}\right)^{1-\beta} \int_{0}^{\infty} e^{-\sigma|1-i y|^{-1}} e^{i \sigma y|1-i y|^{-1}} \sigma^{-\beta}\left(\hat{h}_{u, \theta}(\sigma)-1\right) d \sigma \\
& +\left(\frac{1-i y}{|1-i y|}\right)^{1-\beta} \int_{0}^{\infty} e^{-\sigma|1-i y|^{-1}} e^{i \sigma y|1-i y|^{-1}} \sigma^{-\beta} \hat{h}_{u, \theta}(\sigma)\left(g_{u, \theta}(\sigma /|u-i \theta|)-1\right) d \sigma \\
= & I_{1}+\left(\frac{1-i y}{|1-i y|}\right)^{1-\beta}\left(I_{2}+I_{3}\right) .
\end{aligned}
$$

By Proposition B.1, we have $I_{1}=\Gamma(1-\beta)$. It remains to estimate $I_{2}$ and $I_{3}$.

We show that $\lim _{u, \theta \rightarrow 0} I_{2}=0$ by applying the dominated convergence theorem (DCT). By Potter's bounds (see e.g. [9]), for any fixed $\delta>0,\left|\hat{h}_{u, \theta}(\sigma)\right| \ll \sigma^{\delta}+\sigma^{-\delta}$, for all $\sigma \in(0, \infty)$. Since $|1-i y| \in[1, \sqrt{2}]$, the integrand of $I_{2}$ is bounded, up to a constant, by the function $e^{-\sigma / \sqrt{2}}\left(\sigma^{-(\beta-\delta)}+\sigma^{-(\beta+\delta)}\right)$, which is $L^{1}$ when taking $\delta \in(0,1-\beta)$. Moreover, for each fixed $\sigma \in(0, \infty), \lim _{u, \theta \rightarrow 0} \hat{h}_{u, \theta}(\sigma)=1$ by definition of $\ell$ being slowly varying. Thus, for each $\sigma$,

$$
\lim _{u, \theta \rightarrow 0} \frac{e^{-\sigma|1-i y|^{-1}} e^{i \sigma y|1-i y|^{-1}}}{\sigma^{\beta}}\left(\hat{h}_{u, \theta}(\sigma)-1\right)=0 .
$$

It follows from the DCT that $\lim _{u, \theta \rightarrow 0} I_{2}=0$.

Next, since for any $\delta>0,\left|\hat{h}_{u, \theta}(\sigma)\right| \ll \sigma^{\delta}+\sigma^{-\delta}$, for all $\sigma \in(0, \infty)$ and $\mid 1-$ $i y \mid \in[1, \sqrt{2}]$, we have that $\left|I_{3}\right| \ll\left|g_{u, \theta}-1\right|_{\infty} \int_{0}^{\infty} e^{-\sigma / \sqrt{2}}\left(\sigma^{-(\beta-\delta)}+\sigma^{-(\beta+\delta)}\right) d \sigma \ll$ $\left|g_{u, \theta}-1\right|_{\infty} \rightarrow 0$, as $u, \theta \rightarrow 0$. This ends the proof in case (i).

In case (ii), we write $\hat{v}_{z}=1+\hat{v}_{z}^{1}-\hat{v}_{z}^{2}+i \hat{v}_{z}^{3}-i \hat{v}_{z}^{4}$, where $\hat{v}_{z}^{j} \geq 0$ and $\sup _{x}\left|\hat{v}_{z}^{j}(x)\right|=o(1)$ as $z \rightarrow 1$, for $j=1,2,3,4$.

Define $\hat{G}_{z}^{j}$ such that $d \hat{G}_{z}^{j}=\hat{v}_{z}^{j} d G$. Integrating by parts,

$$
1-\lambda(z)=(u-i \theta) \int_{0}^{\infty}\left(1+\sum_{j=1}^{4} q_{j} \hat{g}_{u, \theta}^{j}(x)\right) e^{-(u-i \theta) x}(1-G(x)) d x
$$

so we obtain

$$
J(z)=\int_{0}^{\infty} E_{u, \theta}(x) h_{u, \theta}(x) d x+\sum_{j=1}^{4} q_{j} \int_{0}^{\infty} E_{u, \theta}(x) \hat{g}_{u, \theta}^{j}(x) h_{u, \theta}(x) d x=I+\sum_{j=1}^{4} q_{j} I^{j}
$$

where $E_{u, \theta}(x):=e^{-(u-i \theta) x}[(u-i \theta) x]^{-\beta}(u-i \theta), q_{1}=1, q_{2}=-1, q_{3}=i, q_{4}=-i$ and $\sup _{x}\left|\hat{g}_{u, \theta}^{j}(x)\right|=o(1)$ as $u, \theta \rightarrow 0$ for $j=1,2,3,4$. Write

$$
I=\int_{0}^{b / \theta} E_{u, \theta}(x) d x+\int_{0}^{b / \theta} E_{u, \theta}(x)\left(h_{u, \theta}(x)-1\right) d x+\int_{b / \theta}^{\infty} E_{u, \theta}(x) h_{u, \theta}(x) d x,
$$


for some positive large $b$. Similarly, for $j=1,2,3,4$, write

$$
I^{j}=\int_{0}^{b / \theta} E_{u, \theta}(x) h_{u, \theta}(x) \hat{g}_{u, \theta}^{j}(x) d x+\int_{b / \theta}^{\infty} E_{u, \theta}(x) h_{u, \theta}(x) \hat{g}_{u, \theta}^{j}(x) d x .
$$

Next, put $y=u / \theta$. Using the substitution $\sigma=|u-i \theta| x$, we have

$$
\begin{aligned}
& \int_{0}^{b / \theta} E_{u, \theta}(x)\left(h_{u, \theta}(x)-1\right) d x \\
& \quad=\left(\frac{y-i}{|y-i|}\right)^{1-\beta} \int_{0}^{|y-i| b}\left(\hat{h}_{u, \theta}(\sigma)-1\right) e^{-\sigma y|y-i|^{-1}} e^{i \sigma|y-i|^{-1}} \sigma^{-\beta} d \sigma
\end{aligned}
$$

and

$$
\begin{aligned}
& \int_{0}^{b / \theta} E_{u, \theta}(x) h_{u, \theta}(x) \hat{g}_{u, \theta}^{j}(x) d x \\
& \quad=\left(\frac{y-i}{|y-i|}\right)^{1-\beta} \int_{0}^{|y-i| b} e^{-\sigma y|y-i|^{-1}} e^{i \sigma|y-i|^{-1}} \sigma^{-\beta} \hat{h}_{u, \theta}(\sigma) \hat{g}_{u, \theta}^{j}\left(\frac{\sigma}{|u-i \theta|}\right) d \sigma .
\end{aligned}
$$

By the argument used for $I_{2}$ in case (i), we have that $\lim _{u, \theta \rightarrow 0} \int_{0}^{b / \theta} E_{u, \theta}(x)\left(h_{u, \theta}(x)-\right.$ 1) $d x=0$. Since $\hat{g}_{u, \theta}^{j}(\sigma /|u-i \theta|) \rightarrow 0$ uniformly in $\sigma$, the argument used for $I_{3}$ in case (i) shows that $\lim _{u, \theta \rightarrow 0} \int_{0}^{b / \theta} E_{u, \theta}(x) h_{u, \theta}(x) \hat{g}_{u, \theta}^{j}(x) d x=0$.

The next step is to estimate

$$
I_{b}^{\infty}:=\int_{b / \theta}^{\infty} E_{u, \theta}(x) h_{u, \theta}(x) d x+\sum_{j=1}^{4} q_{j} \int_{b / \theta}^{\infty} E_{u, \theta}(x) h_{u, \theta}(x) \hat{g}_{u, \theta}^{j}(x) d x .
$$

The substitution $\sigma=\theta x$ gives

$I_{b}^{\infty}=(y-i)^{1-\beta}\left(\int_{b}^{\infty} \frac{e^{-\sigma y} e^{i \sigma}}{\sigma^{\beta}} h_{u, \theta}(\sigma / \theta) d \sigma+\sum_{j=1}^{4} q_{j} \int_{b}^{\infty} \frac{e^{-\sigma y} e^{i \sigma}}{\sigma^{\beta}} h_{u, \theta}(\sigma / \theta) \hat{g}_{u, \theta}^{j}(\sigma / \theta) d \sigma\right)$.

But all the integrals are oscillatory with $\sigma \mapsto e^{-\sigma y} \sigma^{-\beta} h_{u, \theta}(\sigma / \theta)$ and $\sigma \mapsto$ $e^{-\sigma y} \sigma^{-\beta} h_{u, \theta}(\sigma / \theta) \hat{g}_{u, \theta}^{j}(\sigma / \theta)$, respectively, decreasing for each fixed value of $u$ and $\theta$. By Potter's bounds, $h_{u, \theta}(\sigma / \theta) \ll(\sigma|y-i|)^{\delta}+(\sigma|y-i|)^{-\delta} \ll \sigma^{\delta}$ and so $I_{b}^{\infty}=O\left(b^{-(\beta-\delta)}\right)$.

Putting all these together, $\lim _{u, \theta \rightarrow 0} J(z)=\lim _{u, \theta \rightarrow 0} \int_{0}^{b / \theta} E_{u, \theta}(x) d x+O\left(b^{-(\beta-\delta)}\right)$. By Proposition B.1, $\int_{0}^{b / \theta} E_{u, \theta}(x) d x=\Gamma(1-\beta)+O\left(b^{-\beta}\right)$. This ends the proof in case (ii), since $b$ is arbitrary.

Next, we turn to the higher order expansions. The following consequence of (H1) is standard (see for instance [31, Proposition 2.7]). 
Proposition A.2 Assume (H1) and that $\mu(\varphi>n)=\ell(n) n^{-\beta}$ where $\ell$ is slowly varying and $\beta \in[0,1)$. Then there is a constant $C>0$ such that $\| R\left(r e^{i(\theta+h)}\right)-$ $R\left(r e^{i \theta}\right) \| \leq C \ell(1 / h) h^{\beta}$ and $\|R(r)-R(1)\| \leq C \ell\left(\frac{1}{1-r}\right)(1-r)^{\beta}$ for all $\theta \in[0,2 \pi)$, $r \in(0,1], h>0$.

Corollary A.3 The estimates for $R(z)$ in Proposition A.2 are inherited by the families $P(z), Q(z), \lambda(z)$ and $v(z)$, where defined.

Lemma A.4 Assume (H1) and (H2) and let $\beta \in(0,1)$. Suppose that $\mu(\varphi>n)=$ $c\left(n^{-\beta}+H(n)\right)$, where $c>0$ and $H(n)=O\left(n^{-q}\right), q>\beta$. If $q \leq 1$, we assume further that $H(n)=b(n)+c(n)$, where $b$ is monotone with $|b(n)|=O\left(n^{-q}\right)$ and $c(n)$ is summable.

If $q>1$, set $c_{H}=-\Gamma(1-\beta)^{-1} \int_{0}^{\infty} H_{1}(x) d x$ where $H_{1}(x)=[x]^{-\beta}-x^{-\beta}+H([x])$. If $q \leq 1$, set $c_{H}=0$.

Then writing $z=e^{-u+i \theta}, u>0$,

$$
1-\lambda(z)=c \Gamma(1-\beta)\left\{(u-i \theta)^{\beta}-c_{H}(u-i \theta)+O\left(|u-i \theta|^{2 \beta}\right)+D(z),\right.
$$

where $D(z)=O\left(|u-i \theta|^{q}\right)$ if $q \neq 1$, and $D(z)=O\left(|u-i \theta| \log \frac{1}{|u-i \theta|}\right)$ if $q=1$.

Proof We may suppose without loss that $q<\beta+1$. The proof adapts the proof of Lemma 2.4, noting that $\ell=c+o(1)$. The notation for the functions $G$ and $g_{u, \theta}$ used in the proof of Lemma 2.4 keeps its meaning in the proof below. However, since (H1) holds and $1-G(x)=O\left(x^{-\beta}\right)$, we have the more precise estimate $\sup _{x \geq 0}\left|g_{u, \theta}(x)-1\right| \ll$ $|u-i \theta|^{\beta}$. Note also that $1-G(x)=c\left(x^{-\beta}+H_{1}(x)\right)$ where $H_{1}(x)=H(x)+O\left(x^{-(\beta+1)}\right)=$ $O\left(x^{-q}\right)$. As in the proof of Lemma 2.4, we consider separately each of two possible cases: (i) $0 \leq \theta \leq u$, (ii) $0 \leq u \leq \theta$.

In case (i), we have

$$
\begin{aligned}
1-\lambda(z) & =c(u-i \theta)^{\beta} \int_{0}^{\infty} \frac{e^{-(u-i \theta) x}}{[(u-i \theta) x]^{\beta}}(u-i \theta) d x+c(u-i \theta) \int_{0}^{\infty} e^{-(u-i \theta) x} H_{1}(x) d x \\
& +(u-i \theta) \int_{0}^{\infty} e^{-(u-i \theta) x}(1-G(x))\left(g_{u, \theta}(x)-1\right) d x \\
& =c(u-i \theta)^{\beta} I_{1}+c(u-i \theta) I_{2}+(u-i \theta) I_{3} .
\end{aligned}
$$

We already know that $I_{1}=\Gamma(1-\beta)$. Next, we estimate $I_{3}$ proceeding as for $I_{3}$ of case (i) in the proof of Lemma 2.4. Since we are in case (i), $\left|g_{u, \theta}\right| \ll u^{\beta}$, so

$$
\left|(u-i \theta) I_{3}\right| \ll u\left|g_{u, \theta}-1\right|_{\infty} \int_{0}^{\infty} e^{-u x} x^{-\beta} d \sigma \ll u^{2 \beta} \int_{0}^{\infty} e^{-\sigma} \sigma^{-\beta} d \sigma \ll|u-i \theta|^{2 \beta} .
$$

If $q<1$, then similarly

$$
\left|(u-i \theta) I_{2}\right| \ll u \int_{0}^{\infty} e^{-u x} x^{-q} d x=u^{q} \int_{0}^{\infty} e^{-\sigma} \sigma^{-q} d \sigma \ll|u-i \theta|^{q} .
$$


If $q=1$, then

$$
\begin{aligned}
\left|(u-i \theta) I_{2}\right| & \ll u+u \int_{1}^{\infty} e^{-u x} x^{-1} d x=u+u \int_{u}^{\infty} e^{-\sigma} \sigma^{-1} d \sigma \ll u \log \frac{1}{u} \\
& \leq|u-i \theta| \log \frac{1}{|u-i \theta|} .
\end{aligned}
$$

Finally, for $q>1$,

$$
I_{2}=\int_{0}^{\infty}\left(e^{-(u-i \theta) x}-1\right) H_{1}(x) d x+\int_{0}^{\infty} H_{1}(x) d x=I_{2}^{\prime}-c_{H} \Gamma(1-\beta),
$$

where

$$
\left|I_{2}^{\prime}\right| \ll\left(\int_{0}^{1 /|u-i \theta|}|u-i \theta| x^{1-q} d x+\int_{1 /|u-i \theta|}^{\infty} 1 / x^{q} d x\right) \ll|u-i \theta|^{q-1} .
$$

In case (ii), we write

$$
\begin{aligned}
1-\lambda(z) & =c(u-i \theta)^{\beta} \int_{0}^{\infty} \frac{e^{-(u-i \theta) x}}{[(u-i \theta) x]^{\beta}}(u-i \theta) d x+c(u-i \theta) \int_{0}^{\infty} e^{-(u-i \theta) x} H_{1}(x) d x \\
& +(u-i \theta) \sum_{j=1}^{4} q_{j} \int_{0}^{\infty} e^{-(u-i \theta) x}(1-G(x)) \hat{g}_{u, \theta}^{j}(x) d x \\
& =(u-i \theta)^{\beta} I_{1}+(u-i \theta) I_{2}+(u-i \theta) \sum_{j=1}^{4} q_{j} I^{j},
\end{aligned}
$$

where $q_{1}=1, q_{2}=-1, q_{3}=i, q_{4}=-i$ and $\sup _{x}\left|\hat{g}_{u, \theta}^{j}(x)\right| \ll|u-i \theta|^{\beta}$ for $j=1,2,3,4$.

Again $I_{1}=\Gamma(1-\beta)$. To estimate $I^{j}, j=1,2,3$, 4 , we proceed similarly to the case (ii) of the proof of Lemma 2.4. Put $y=u / \theta$, so $y \leq 1$. Substituting $\sigma=\theta x$,

$$
\theta I^{j}=\int_{0}^{\infty} e^{-\sigma y} e^{i \sigma}(1-G(\sigma / \theta)) \hat{g}_{u, \theta}^{j}(\sigma / \theta) d \sigma .
$$

Since $\sigma \mapsto e^{-\sigma y}(1-G(\sigma / \theta)) \hat{g}_{u, \theta}^{j}(\sigma / \theta)$ is decreasing for each fixed value of $u$ and $\theta$,

$$
\begin{aligned}
\int_{0}^{\infty} e^{-\sigma y} \cos \sigma(1-G(\sigma / \theta)) \hat{g}_{u, \theta}^{j}(\sigma / \theta) d \sigma & \leq \int_{0}^{\pi / 2} e^{-\sigma y} \cos \sigma(1-G(\sigma / \theta)) \hat{g}_{u, \theta}^{j}(\sigma / \theta) d \sigma \\
& \ll \theta^{2 \beta} \int_{0}^{\pi / 2} \sigma^{-\beta} d \sigma \ll \theta^{2 \beta} .
\end{aligned}
$$

The integral with cos replaced by sin can be treated similarly, so $\left|(u-i \theta) I^{j}\right| \ll$ $\theta\left|I_{j}\right| \ll \theta^{2 \beta} \leq|u-i \theta|^{2 \beta}$. 
It remains to estimate $I_{2}$. If $q<1$, define $H_{2}(x)=[x]^{-\beta}-x^{-\beta}+c([x])$. Then

$$
I_{2}=-\int_{0}^{\infty} e^{-(u-i \theta) x} b([x]) d x+\int_{0}^{\infty} e^{-(u-i \theta) x} H_{2}(x) d x=-I_{2}^{\prime}+I_{2}^{\prime \prime} .
$$

Clearly, $I_{2}^{\prime \prime}=O(1)$ since $H_{2}$ is integrable. Let $y=u / \theta$, so $y \leq 1$. Substituting $\sigma=\theta x$,

$$
\theta I_{2}^{\prime}=\int_{0}^{\infty} e^{-\sigma y} e^{i \sigma} b([\sigma / \theta]) d \sigma
$$

Suppose for definiteness that $b$ is positive and decreasing. Since $\sigma \mapsto e^{-\sigma y} b([\sigma / \theta])$ is decreasing for each fixed value of $u$ and $\theta$, we have

$$
\int_{0}^{\infty} e^{-\sigma y} \cos \sigma b([\sigma / \theta]) d \sigma \leq \int_{0}^{\pi / 2} e^{-\sigma y} \cos \sigma b([\sigma / \theta]) d \sigma \ll \theta^{q} \int_{0}^{\pi / 2} \sigma^{q} d \sigma \ll \theta^{q} .
$$

The integral with cos replaced by sin is treated similarly. Hence $\left|(u-i \theta) I_{2}\right| \ll \theta\left|I_{2}\right| \ll$ $|u-i \theta|^{q}$. The proof for $q=1$ is identical, except that in the last step

$$
\int_{0}^{\infty} e^{-\sigma y} \cos \sigma b([\sigma / \theta]) d \sigma \leq \theta+\theta \int_{\theta}^{\pi / 2} \sigma^{-1} d \sigma \ll \theta \log \frac{1}{\theta} \ll|u-i \theta| \log \frac{1}{|u-i \theta|} .
$$

If $q>1$, then we proceed as in case (i).

Proof of Lemma 2.5 Taking $q=2 \beta$, it follows from Lemma A.4 that $(1-\lambda(z))^{-1} P$ has the desired expansion. By (A.1), Corollary A.3 and Proposition A.1, $\| T(z)-(1-$ $\lambda(z))^{-1} P \|=O(1)$.

Proof of Lemma 2.7 The basic argument is similar to the one used in the proof of Lemma 2.5, simplified by the fact that the various integrals are absolutely convergent. We give the details for the more difficult case (b). For notational convenience, we write $\lambda(u)$ instead of $\lambda\left(e^{-u}\right)$.

Let $G(x)=\mu(\varphi \leq x)$ so that $1-G(x)=\ell(x)^{-1}+H(x)$, where $\ell \in O \Pi_{\hat{\ell}}$ and $H(x)=O\left(\ell(x)^{-2} \hat{\ell}(x)\right)$. Write $\lambda(u)=1+\int_{0}^{\infty}\left(e^{-u x}-1\right) \hat{v}_{u}(x) d G(x)$, where $\left|\hat{v}_{u}-1\right|_{\infty} \ll$ $\ell(1 / u)^{-1}$ as $u \rightarrow 0$. Put $d \hat{G}_{u}=\hat{v}_{u} d G$. Integrating by parts,

$$
\begin{aligned}
1-\lambda(u) & =u \int_{0}^{\infty} e^{-u x} g_{u}(x)(1-G(x)) d x \\
& =u \int_{0}^{\infty} e^{-u x} g_{u}(x) \frac{1}{\ell(x)} d x+u \int_{0}^{\infty} e^{-u x} g_{u}(x) H(x) d x
\end{aligned}
$$

where $\left|g_{u}(x)-1\right|_{\infty} \ll \ell(1 / u)^{-1}$ as $u \rightarrow 0$. Thus,

$$
\begin{aligned}
1-\lambda(u) & =\frac{1}{\ell(1 / u)} \int_{0}^{\infty} e^{-\sigma} \frac{\ell(1 / u)}{\ell(\sigma / u)} d \sigma+\int_{0}^{\infty} e^{-\sigma}\left(g_{u}(\sigma / u)-1\right) \frac{1}{\ell(\sigma / u)} d \sigma \\
& +\int_{0}^{\infty} e^{-\sigma} g_{u}(\sigma / u) H(\sigma / u) d \sigma=I_{1}+I_{2}+I_{3} .
\end{aligned}
$$


Now,

$$
\begin{aligned}
I_{1} & =\frac{1}{\ell(1 / u)} \int_{0}^{\infty} e^{-\sigma} d \sigma+\frac{1}{\ell(1 / u)} \int_{0}^{\infty} e^{-\sigma}\left(\frac{\ell(1 / u)}{\ell(\sigma / u)}-1\right) d \sigma \\
& =\frac{1}{\ell(1 / u)}+\frac{\hat{\ell}(1 / u)}{\ell(1 / u)^{2}} \int_{0}^{\infty} e^{-\sigma} \frac{\ell(1 / u)}{\ell(\sigma / u)}\left(\frac{\ell(1 / u)-\ell(\sigma / u)}{\hat{\ell}(1 / u)}\right) d \sigma
\end{aligned}
$$

By Potter's bounds, for any fixed $\delta>0, \ell(1 / u) \ell(\sigma / u)^{-1} \ll \sigma^{\delta}+\sigma^{-\delta}$. Also, by [9, Theorem 3.8.6] (which is the analogue of Potter's bounds for de Haan functions), for any fixed $\delta>0, \hat{\ell}^{-1}(1 / u)|\ell(1 / u)-\ell(\sigma / u)| \ll \sigma^{\delta}+\sigma^{-\delta}$. Hence,

$$
I_{1}=\frac{1}{\ell(1 / u)}+O\left(\frac{\hat{\ell}(1 / u)}{\ell(1 / u)^{2}}\right) \text {. }
$$

Next,

$$
\left|I_{2}\right| \ll \frac{1}{\ell(1 / u)} \int_{0}^{\infty} e^{-\sigma} \frac{1}{\ell(\sigma / u)} d \sigma \ll \frac{1}{\ell(1 / u)^{2}} \int_{0}^{\infty} e^{-\sigma} \frac{\ell(1 / u)}{\ell(\sigma / u)} d \sigma \ll \frac{1}{\ell(1 / u)^{2}},
$$

and similarly $\left|I_{3}\right|=O\left(\ell(1 / u)^{-2} \hat{\ell}(1 / u)\right)$. Putting all these together, we have shown that $1-\lambda(u)=\ell(1 / u)^{-1}+O\left(\ell(1 / u)^{-2} \hat{\ell}(1 / u)\right)$. Hence

$$
(1-\lambda(u))^{-1}=\ell(1 / u)+O(\hat{\ell}(1 / u)) .
$$

Again, the result follows by A.1 , Corollary A.3 and Proposition A.1.

\section{B Some contour integrals}

Proposition B.1 Let $\beta \in(0,1)$. Then for every $u>0$ and $\theta \neq 0$ fixed, we have

$$
\int_{0}^{R} \frac{e^{-(u-i \theta) x}}{[(u-i \theta) x]^{\beta}}(u-i \theta) d x=\Gamma(1-\beta)+O\left(R^{-\beta}\right) \text {, as } R \rightarrow \infty .
$$

Proof Write

$$
\int_{0}^{R} e^{-(u-i \theta) x}[(u-i \theta) x]^{-\beta}(u-i \theta) d x=\int_{\Gamma} e^{-w} w^{-\beta} d w
$$

where $\Gamma$ is a line segment of length $R$ emanating from 0 in the fourth quadrant. The angle formed by $\Gamma$ with the positive real axis is $\phi:=-\arg (u-i \theta) \in[0, \pi / 2]$.

Define the $\operatorname{arcs} S_{\delta}=\left\{\delta e^{i \psi}:-\phi \leq \psi \leq 0\right\}, S_{R}=\left\{R e^{i \psi}:-\phi \leq \psi \leq 0\right\}$, and let $L$ be the line segment from $\delta$ to $R$ along the real axis. By Cauchy's theorem,

$$
\int_{\Gamma} e^{-w} w^{-\beta} d w=\lim _{\delta \rightarrow 0}\left(\int_{S_{\delta}}+\int_{L}-\int_{S_{R}}\right) e^{-w} w^{-\beta} d w
$$


On $S_{\delta}$

$$
\left|\int_{S_{\delta}} e^{-w} w^{-\beta} d w\right|=\left|\int_{-\phi}^{0} e^{-\delta e^{i \psi}}\left(\delta e^{i \psi}\right)^{-\beta} \delta i e^{i \psi} d \psi\right| \leq \int_{-\pi / 2}^{0} \delta^{1-\beta} d \psi \leq(\pi / 2) \delta^{1-\beta} .
$$

On $S_{R}$

$$
\left|\int_{S_{R}} e^{-w} w^{-\beta} d w\right|=\left|\int_{-\phi}^{0} e^{-R e^{-\psi}}\left(R e^{i \psi}\right)^{-\beta} \operatorname{Ri} e^{i \psi} d \psi\right| \leq R^{1-\beta} \int_{0}^{\pi / 2} e^{-R \cos \psi} d \psi
$$

Since $\cos \psi>1-2 \psi / \pi$ for $\psi \in(0, \pi / 2)$ (just draw the graph of $\cos \psi$ and $1-2 \psi / \pi$ ),

$$
\left|\int_{S_{R}} e^{-w} w^{-\beta} d w\right| \leq R^{1-\beta} \int_{0}^{\pi / 2} e^{-R(1-2 \psi / \pi)} d \psi=\frac{\pi}{2 R^{\beta}}\left(1-e^{-R}\right) .
$$

Also, $\int_{L} e^{-w} w^{-\beta} d w=\int_{\delta}^{R} e^{-t} t^{-\beta} d t$. Thus, letting $\delta \rightarrow 0, \int_{\Gamma} e^{-w} w^{-\beta} d w=$ $\int_{0}^{R} e^{-t} t^{-\beta} d t+O\left(R^{-\beta}\right)=\int_{0}^{\infty} e^{-t} t^{-\beta} d t+O\left(R^{-\beta}\right)$, which ends the proof.

Proposition B.2 Let $\beta \in(0,1)$. Then

$$
\int_{-\infty}^{\infty} \frac{1}{(1-i \sigma)^{\beta+1}} e^{-i \sigma} d \sigma=\frac{2 \pi}{e} \frac{1}{\Gamma(1+\beta)}
$$

Proof First, write

$$
\int_{-\infty}^{\infty} \frac{1}{(1-i \sigma)^{\beta+1}} e^{-i \sigma} d \sigma=\frac{i}{e} \int_{\gamma} \frac{e^{w}}{w^{\beta+1}} d w
$$

where the contour $\gamma=\{\operatorname{Re} w=1\}$ is traversed downwards.

We estimate $\int_{\gamma} e^{w} w^{-\beta} d w$ and then integrate by parts to complete the proof. The diagram below shows a simple closed contour consisting of $\gamma$ together with oriented curves $C, L^{ \pm}, M^{ \pm}, S^{ \pm}$

By Cauchy's theorem,

$$
\int_{\gamma} \frac{e^{w}}{w^{\beta}} d w=\lim _{R \rightarrow \infty} \lim _{\delta \rightarrow 0^{+}}\left(-\int_{M^{+}}+\int_{S^{+}}+\int_{L^{+}}-\int_{C}-\int_{L^{-}}-\int_{S^{-}}+\int_{M^{-}}\right) \frac{e^{w}}{w^{\beta}} d w .
$$

Now $C=\left\{w=\delta e^{i \theta}: \theta \in(-\pi / 2, \pi / 2)\right\}$, and so

$$
\left|\int_{C} e^{w} w^{-\beta} d w\right|=\left|\int_{-\pi / 2}^{\pi / 2} \exp \left(\delta e^{i \theta}\right) \delta^{1-\beta} e^{i \theta(1-\beta)} d \theta\right| \ll \delta^{1-\beta}
$$

On $M^{ \pm}$, we have

$$
\left|\int_{M^{ \pm}} e^{w} w^{-\beta} d w\right|=\left|\int_{0}^{1} e^{t} e^{i R}(t \pm i R)^{-\beta} d t\right| \leq R^{-\beta} \int_{0}^{1} e^{t} d t \ll R^{-\beta} .
$$




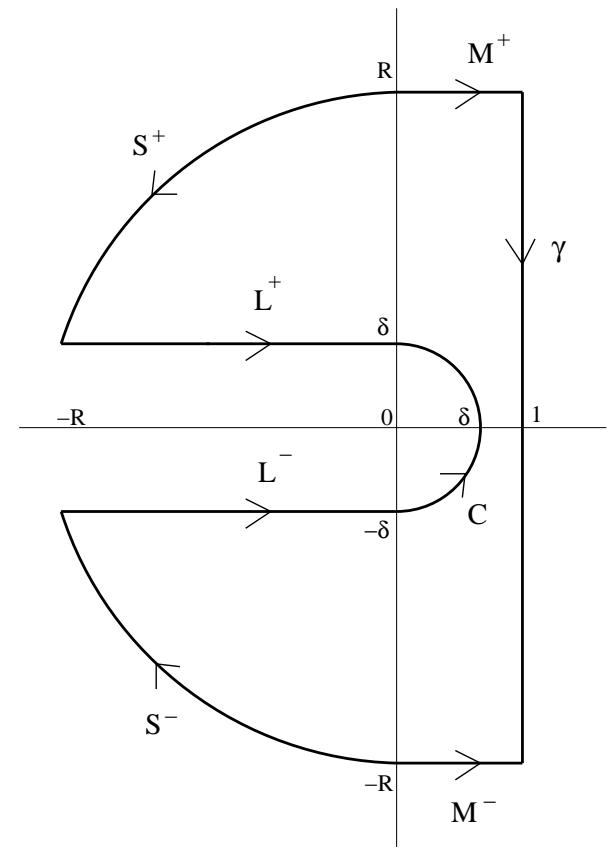

On $S^{ \pm}$, we write

$$
\begin{aligned}
\left|\int_{S^{ \pm}} e^{w} w^{-\beta} d w\right| & =\left|\int_{\pi / 2}^{\pi-\epsilon} e^{R e^{ \pm i \theta}}\left(R e^{ \pm i \theta}\right)^{-\beta} R i e^{ \pm i \theta} d \theta\right| \leq R^{1-\beta} \int_{\pi / 2}^{\pi} e^{R \cos \theta} d \theta \\
& =R^{1-\beta} \int_{0}^{\pi / 2} e^{-R \sin \theta} d \theta .
\end{aligned}
$$

(Here, $\epsilon=\epsilon(R, \delta)$ is chosen so that $S^{ \pm}$connects with $L^{ \pm}$as shown.) But $\sin \theta>\theta / \pi$ for $\theta \in(0, \pi / 2)$. Hence, $\left|\int_{S^{ \pm}} e^{w} w^{-\beta} d w\right| \leq R^{1-\beta} \int_{0}^{\pi / 2} e^{-R \theta / \pi} d \theta=\pi R^{-\beta}\left(1-e^{-R / 2}\right)$.

We have shown that $\int_{\gamma} \frac{e^{w}}{w^{\beta}} d w=\lim _{R \rightarrow \infty} \lim _{\delta \rightarrow 0^{+}}\left(\int_{L^{+}} \frac{e^{w}}{w^{\beta}} d w-\int_{L^{-}} \frac{e^{w}}{w^{\beta}} d w\right)$. Now

$$
\int_{L^{ \pm}} e^{w} w^{-\beta} d w=\int_{-R}^{0} e^{t} e^{ \pm i \delta}\left(t^{2}+\delta^{2}\right)^{-\beta / 2} e^{-i \beta \arg (t \pm i \delta)} d t .
$$

Since $t<0, \lim _{\delta \rightarrow 0^{+}} \arg (t \pm i \delta) \rightarrow \pm \pi$. Hence, the integrand converges pointwise to $e^{\mp i \pi \beta} e^{t}|t|^{-\beta}$ as $\delta \rightarrow 0^{+}$. Moreover, the integrand is bounded by $e^{t}|t|^{-\beta}$ which is integrable on $[-R, 0]$. It follows from the DCT that $\lim _{\delta \rightarrow 0^{+}} \int_{L^{ \pm}} e^{w} w^{-\beta} d w=$ $e^{\mp i \pi \beta} \int_{-R}^{0} e^{t}|t|^{-\beta} d t=e^{\mp i \pi \beta} \int_{0}^{R} e^{-t} t^{-\beta} d t$. Hence,

$$
\int_{\gamma} \frac{e^{w}}{w^{\beta}} d w=-2 i \sin \pi \beta \lim _{R \rightarrow \infty} \int_{0}^{R} e^{-t} t^{-\beta} d t=-2 \pi i \frac{1}{\Gamma(\beta)},
$$

where we have used the formula $\frac{\sin \pi \beta}{\pi}=\frac{1}{\Gamma(1-\beta) \Gamma(\beta)}$. 
Finally,

$$
\frac{i}{e} \int_{\gamma} \frac{e^{w}}{w^{\beta+1}} d w=-\left.\frac{i}{e \beta} \frac{e^{w}}{w^{\beta}}\right|_{w=1-i \sigma, \sigma \rightarrow-\infty} ^{w=1-i \sigma, \sigma \rightarrow \infty}+\frac{i}{e \beta} \int_{\gamma} \frac{e^{w}}{w^{\beta}}=\frac{2 \pi}{e \beta} \frac{1}{\Gamma(\beta)},
$$

as required.

Corollary B.3 For any $\rho>0 \gamma \in(0,1)$,

$$
\int_{-1 / n^{\gamma}}^{1 / n^{\gamma}} \frac{e^{-i n \theta}}{\left(\frac{1}{n}-i \theta\right)^{\rho+1}} d \theta=\frac{2 \pi}{e} \frac{n^{\rho}}{\Gamma(1+\rho)}+O\left(n^{\rho \gamma}\right)
$$

Proof Compute that

$$
\begin{aligned}
\int_{-1 / n^{\gamma}}^{1 / n^{\gamma}} \frac{e^{-i n \theta}}{\left(\frac{1}{n}-i \theta\right)^{\rho+1}} d \theta & =n^{\rho} \int_{-n^{1-\gamma}}^{n^{1-\gamma}} \frac{e^{-i \sigma}}{(1-i \sigma)^{\rho+1}} d \sigma \\
& =n^{\rho} \int_{-\infty}^{\infty} \frac{e^{-i \sigma}}{(1-i \sigma)^{\rho+1}} d \sigma-n^{\rho}\left(\int_{-\infty}^{-n^{1-\gamma}}+\int_{n^{1-\gamma}}^{\infty}\right) \frac{e^{-i \sigma}}{(1-i \sigma)^{\rho+1}} d \sigma \\
& =\frac{2 \pi}{e} \frac{n^{\rho}}{\Gamma(1+\rho)}+O\left(n^{\rho} \int_{n^{1-\gamma}}^{\infty} \frac{1}{\sigma^{\rho+1}} d \sigma\right) \\
& =\frac{2 \pi}{e} \frac{n^{\rho}}{\Gamma(1+\rho)}+O\left(n^{\rho \gamma}\right)
\end{aligned}
$$

where we have used Proposition B.2.

\section{Tail sequences for (1.1) and (1.2)}

The following proposition is an improved version of [31, Proposition 11.9]. Recall that $h$ denotes the density for the measure $\mu$.

Proposition C.1 Suppose that $f:[0,1] \rightarrow[0,1]$ is given as in (1.1) with $\beta=1 / \alpha \in$ $(0,1)$. Let $C$ be a compact subset of $(0,1]$. Then there exists $Y \subset(0,1]$ compact with $C \subset Y$, such that the first return function $\varphi: Y \rightarrow \mathbb{Z}^{+}$satisfies $\mu(\varphi>n)=$ $c n^{-\beta}-b(n)+O\left(n^{-(\beta+1)}\right)$, where $c=\frac{1}{4} \beta^{\beta} h\left(\frac{1}{2}\right)$ and $b(n)$ is a decreasing function satisfying $b(n)=O\left(n^{-2 \beta}\right)$.

Proof First, let $Y=\left[\frac{1}{2}, 1\right]$. Let $x_{n} \in\left(0, \frac{1}{2}\right]$ be the sequence with $x_{1}=\frac{1}{2}$ and $x_{n}=f x_{n+1}$ so $x_{n} \rightarrow 0$. It is well known (see for instance [30]) that $x_{n} \sim \frac{1}{2} \beta^{\beta} n^{-\beta}$. Since $x_{n}-x_{n+1}=f x_{n+1}-x_{n+1}=O\left(n^{-(\beta+1)}\right)$, we have $x_{n}=\frac{1}{2} \beta^{\beta} n^{-\beta}+O\left(n^{-(\beta+1)}\right)$.

The density $h$ is globally Lipschitz on $(\epsilon, 1]$ for any $\epsilon>0$ (see for example [21] or [30, Lemma 2.1]). Furthermore, it follows from [39, Lemma 2], see also [32], that $h$ 
is decreasing. Hence for $x \in\left[\frac{1}{2}, 1\right]$ we can write $h(x)=h\left(\frac{1}{2}\right)-\tilde{h}(x)$ where $\tilde{h}$ is positive and Lipschitz.

Set $y_{n}=\frac{1}{2}\left(x_{n}+1\right)\left(\right.$ so $\left.f y_{n}=x_{n}\right)$. Then $\varphi=n$ on $\left[y_{n}, y_{n-1}\right]$, hence $\{\varphi>n\}=$ $\left[\frac{1}{2}, y_{n}\right]$. It follows that

$$
\mu(\varphi>n)=\int_{1 / 2}^{y_{n}} h(x) d x=\frac{1}{2} x_{n} h\left(\frac{1}{2}\right)-b(n)=\frac{1}{4} \beta^{\beta} h\left(\frac{1}{2}\right) n^{-\beta}+O\left(n^{-(1+\beta)}\right)-b(n),
$$

where $b(n)=\int_{1 / 2}^{y_{n}} \tilde{h}(x) d x$ is decreasing. Moreover, $b(n) \ll\left(y_{n}-\frac{1}{2}\right)^{2}=\frac{1}{4} x_{n}^{2} \ll n^{-2 \beta}$.

The same estimates are obtained by inducing on $Y=\left[x_{q}, 1\right]$ for any fixed $q \geq 0$.

Proposition C.2 Suppose that $f:[0,1] \rightarrow[0,1]$ is given as in (1.2). Let $C$ be a compact subset of $(0,1]$. Then there exists $Y \subset(0,1]$ compact with $C \subset Y$, such that the first return function $\varphi: Y \rightarrow \mathbb{Z}^{+}$satisfies $\mu(\varphi>n)=c \log ^{-1} n+O\left(\log ^{-2} n\right)$, where $c=\frac{1}{2} h\left(\frac{1}{2}\right)$.

Proof First, let $Y=\left[\frac{1}{2}, 1\right]$. Let $x_{n} \in\left(0, \frac{1}{2}\right]$ be the sequence with $x_{1}=\frac{1}{2}$ and $x_{n}=f x_{n+1}$ so $x_{n} \rightarrow 0$. We claim that $x_{n} \sim \log ^{-1} n$, in accordance with [37, Remark 2, p. 94]. By (1.2),

$$
\begin{aligned}
e^{1 / x_{j}} & =\exp \left(\frac{1}{x_{j+1}}\left(1+x_{j+1} e^{-1 / x_{j+1}}\right)^{-1}\right) \\
& =\exp \left(\frac{1}{x_{j+1}}\left(1-x_{j+1} e^{-1 / x_{j+1}}+O\left(x_{j+1}^{2} e^{-2 / x_{j+1}}\right)\right)\right) \\
& =e^{1 / x_{j+1}} \exp \left(-e^{-1 / x_{j+1}}+O\left(x_{j+1} e^{-2 / x_{j+1}}\right)\right) \\
& =e^{1 / x_{j+1}}\left(1-e^{-1 / x_{j+1}}+O\left(e^{-2 / x_{j+1}}\right)\right)=e^{1 / x_{j+1}}-1+O\left(e^{-1 / x_{j+1}}\right) .
\end{aligned}
$$

Hence $e^{1 / x_{j+1}}-e^{1 / x_{j}}=1+O\left(e^{-1 / x_{j+1}}\right)$. Summing from $j=1$ to $n-1$,

$$
e^{1 / x_{n}}=e^{1 / x_{1}}+n-1+O\left(\sum_{j=2}^{n} e^{-1 / x_{j}}\right)
$$

Since $x_{n} \rightarrow 0$, for $n$ sufficiently large we have $e^{1 / x_{n}} \in\left(\frac{1}{2} n, \frac{3}{2} n\right)$. Hence $1 / x_{n} \in$ $\left(\log n+\log \frac{1}{2}, \log n+\log \frac{1}{2}\right)$ and $x_{n} \sim \log ^{-1} n$ verifying the claim. Moreover, $x_{n}-x_{n+1}=$ $f x_{n+1}-x_{n+1}=O\left(1 /\left(n \log ^{2} n\right)\right)$, so $x_{n}=\log ^{-1} n+O\left(\left(n \log ^{2} n\right)^{-1}\right)$.

Again, the density $h$ is globally Lipschitz on $(\epsilon, 1]$ for any $\epsilon>0$ (see [37]). Thus, in the notation of the proof of Proposition C.1,

$$
\mu(\varphi>n)=\int_{\frac{1}{2}}^{y_{n}} h(x) d x=\left(y_{n}-\frac{1}{2}\right) h\left(\frac{1}{2}\right)+O\left(y_{n}-\frac{1}{2}\right)^{2}=\frac{1}{2} h\left(\frac{1}{2}\right) \log ^{-1} n+O\left(\log ^{-2} n\right),
$$

as required.

The same estimates are obtained by inducing on $Y=\left[x_{q}, 1\right]$ for any fixed $q \geq 0$. 
Acknowledgements The research of IM and DT was supported in part by EPSRC Grant EP/F031807/1. We wish to thank Roland Zweimüller for useful conversations.

\section{References}

[1] J. Aaronson. Random f-expansions. Ann. Probab. 14 (1986) 1037-1057.

[2] J. Aaronson. An Introduction to Infinite Ergodic Theory. Math. Surveys and Monographs 50, Amer. Math. Soc., 1997.

[3] J. Aaronson and M. Denker. Upper bounds for ergodic sums of infinite measure preserving transformations. Trans. Amer. Math. Soc. 319 (1990) 101-138.

[4] J. Aaronson and M. Denker. Local limit theorems for partial sums of stationary sequences generated by Gibbs-Markov maps. Stoch. Dyn. 1 (2001) 193-237.

[5] J. Aaronson, M. Denker and A. M. Fisher. Second order ergodic theorems for ergodic transformations of infinite measure spaces. Proc. Amer. Math. Soc. 114 (1992), 115-127.

[6] J. Aaronson, M. Denker and M. Urbański. Ergodic theory for Markov fibred systems and parabolic rational maps. Trans. Amer. Math. Soc. 337 (1993) 495548.

[7] S. Aljančić, R. Bojanić and M. Tomić. Slowly varying functions with remainder and their applications in analysis. Serbian Acad. Sci. Acts Monographs 467 (1974), Beograd.

[8] N. H. Bingham. Limit theorems for occupation times of Markoff processes. Z. Wahrscheinlichkeistheorie verw. Geb. 17 (1971) 1-22.

[9] N. H. Bingham, C. M. Goldie and J. L. Teugels. Regular variation. Encyclopedia of Mathematics and its Applications 27, Cambridge University Press, Cambridge, 1987.

[10] D. A. Darling and M. Kac. On occupation times for Markoff processes. Trans. Amer. Math. Soc. 84 (1957) 444-458.

[11] K. B. Erickson. Strong renewal theorems with infinite mean. Trans. Amer. Math. Soc. 151 (1970) 263-291.

[12] W. Feller. An Introduction to Probability Theory and its Applications, II. Wiley, New York, 1966.

[13] G. Freud. Restglied eines Tauberschen Satzes. I. Acta Math. Acad. Sci. Hungar. 2 (1951) 299-308. 
[14] A. Garsia and J. Lamperti. A discrete renewal theorem with infinite mean. Comment. Math. Helv. 37 (1962/1963) 221-234.

[15] S. Gouëzel. Sharp polynomial estimates for the decay of correlations. Israel J. Math. 139 (2004) 29-65.

[16] S. Gouëzel. Berry-Esseen theorem and local limit theorem for non uniformly expanding maps. Ann. Inst. H. Poincaré Probab. Statist. 41 (2005) 997-1024.

[17] J. Geluk. $\pi$ regular variation. Proc. Amer. Math. Soc. 82 (1981) 565-570.

[18] J. Geluk and L. de Haan. On functions with small differences. Indag. Math. 84 (1981) 187-194.

[19] L. de Haan. On Regular Variation and its Application to the Weak Convergence of Sample Extremes. Centre Tract 32 (1970), Amsterdam.

[20] L. de Haan. An Abel Tauber theorem for Laplace transform. J. London Math. Soc 13 (1976) 537-542.

[21] H. Hu. Decay of correlations for piecewise smooth maps with indifferent fixed points. Ergodic Theory Dynam. Systems 24 (2004) 495-524.

[22] A. Ingham. On Tauberian theorems. Proc. London Math. Soc. 14A (1965) 157173.

[23] G. Jordan. Regularly varying functions and convolutions with real kernels. Trans. Amer. Math. Soc. 194 (1974) 177-194.

[24] J. Karamata. Über die Hardy-Littlewoodschen Umkehrungen des Abelschen Stetigkeitssatzes. Math. Z. 32 (1930) 319-320.

[25] J. Karamata. Neuer Beweis und Verallgemeinerung der Tauberschen Sätze, welche die Laplacesche und Stieltjessche Transformation betreffen. J. Reine Angwe. Math. 164 27-39.

[26] J. Korevaar. A very general form of Littlewood's theorem. Indag. Math 16 (1954) $36-45$.

[27] J. Korevaar. Another numerical Tauberian theorem for power series. Indag. Math. 16 (1954) 45-56.

[28] J. Korevaar. Tauberian theory. A century of developments. Springer Verlag Berlin Heidelberg, (2004).

[29] J. Lamperti. An invariance principle in renewal theory. Ann. Math. Statistics. 33 (1962) 685-696. 
[30] C. Liverani, B. Saussol and S. Vaienti. A probabilistic approach to intermittency. Ergodic Theory Dynam. Systems 19 (1999) 671-685.

[31] I. Melbourne and D. Terhesiu. Operator renewal theory and mixing rates for dynamical systems with infinite measure. Preprint 2010.

[32] R. Murray. Ulam's method for some non-uniformly expanding maps. Discrete Cont. Dyn. Syst. 26 (2010) 1007-1018.

[33] E. Omey. Tauberian theorems with remainder. J. London Math. Soc. 32 (1985) 116-132.

[34] Y. Pomeau and P. Manneville. Intermittent transition to turbulence in dissipative dynamical systems. Comm. Math. Phys. 74 (1980) 189-197.

[35] O. M. Sarig. Subexponential decay of correlations. Invent. Math. 150 (2002) 629-653.

[36] M. A. Subhankulov. Tauberian theorems with remainder term. Mat. Sbornik 52(94) (1960) 823-846. Amer. Math. Soc. Transl. 26 (1963) 311-338.

[37] M. Thaler. Transformations on $[0,1]$ with infinite invariant measures. Israel. J. Math. 46 (1983) 67-96

[38] M. Thaler. A limit theorem for the Perron-Frobenius operator of transformations on $[0,1]$ with indifferent fixed points. Israel J. Math. 91 (1995) 111-127.

[39] M. Thaler. A limit theorem for sojourns near indifferent fixed points of onedimensional maps. Ergod. Th. Dynam. Syst. 22 (2002) 1289-1312.

[40] M. Thaler and R. Zweimüller. Distributional limit theorems in infinite ergodic theory. Probab. Theory Related Fields 135 (2006) 15-52.

[41] R. Zweimüller. Ergodic structure and invariant densities of non-Markovian interval maps with indifferent fixed points. Nonlinearity 11 (1998) 1263-1276.

[42] R. Zweimüller. Ergodic properties of infinite measure-preserving interval maps with indifferent fixed points. Ergodic Theory Dynam. Systems 20 (2000) 1519 1549 . 